\title{
Quadrotor Energy-Based Control Laws: A Unit-Quaternion Approach
}

\author{
M.E. Guerrero • H. Abaunza - P. Castillo · R. Lozano - C.D. Garcia
}

Received: date / Accepted: date

\begin{abstract}
This article presents the designs, simulations and real-time experimental results of two energy-based control strategies to stabilize an Unmanned Aerial Vehicles (UAV) using a quaternion representation of the attitude. The mathematical model is based on EulerLagrange formulation using a logarithmic mapping in the quaternion space. The proposed solutions introduce a new approach: a quaternion-energy-based control, which use an energy-based expression defined as a Lyapunov function. The control laws are described with unit quaternions and their axis-angle representation. The proposed algorithms allow the stabilization of the quadrotor in all its states. The strategies ensure the stability of the closed loop system. Simulation results and experimental validations are developed to verify the effectiveness of the proposed controllers.
\end{abstract}

Keywords Energy-Based Control - Quaternion · Quadrotor · Real-time validation · Lyapunov analysis

\section{Introduction}

Unmanned Aerial Vehicles have experienced a significant development in the last years. Various mathematical models and many control strategies based on

M.E. Guerrero, H. Abaunza, P. Castillo and R. Lozano

Sorbonne universités, Université de Technologie de Compiègne, CNRS, Heudiasyc UMR 7253, CS $60 \quad 319$, 60203 Compiègne cedex.

E-mail: (maria-eusebia.guerrero-sanchez, habaunza, castillo, rlozano)@utc.fr

R. Lozano is also with UMI LAFMIA 3175 CINVESTAVCNRS, Mexico.

C.D. Garcia

Department of Electronic Engineering, CENIDET, Mexico.

E-mail: cgarcia@cenidet.edu.mx classic or modern control theory have been developed for these vehicles. Often, in the major part of the literature on quadrotors, Euler angles are used for the attitude parametrization, which is a very natural way of describing orientation. However, this representation presents inherent singularities and many non-linearities by the use of trigonometric functions, which cause extensive representations of the control algorithms and complications in the design of control strategies. For these reasons, the use of a quaternions instead of Euler angles to model the rotational dynamics and to develop control laws for quadrotors is becoming very popular amongst some researchers.

Few works have investigated the quadrotor attitude control problem using a hyper complex number of rank 4 known as quaternions. For example, in [1] an approach that utilizes an attitude parametrization based on quaternions is proposed. The strategy consists of two stages. First an input-output linearization from the altitude position to the thrust is performed, followed by a second input-output linearization from the translational position to the control torques. This separation leads to a so called quasi-static feedback linearization that omits additional controller state. Also, in [2] a hierarchical controller design based on non-linear $H_{\infty}$ theory and backstepping technique is developed for a nonlinear and coupled dynamic attitude system using conventional quaternion based method. The derived controller combines the attractive features of $H_{\infty}$ optimal controller and the advantages of the backstepping technique leading to a control law which avoids winding phenomena.

Similarly, in [3] various control techniques for a quadrotor using a quaternion representation of the attitude 
were designed. All attitude controllers use a quaternion error to compute control signals that are computed from an actual quaternion and a desired quaternion obtained from a position controller. Attitude and position control laws are obtained using a PD, LQR and backstepping control technique. In [4] the attitude stabilization problem for a quadrotor is considered. Using a new Lyapunov function, an exponentially stabilizing controller based upon the compensation of the Coriolis and gyroscopic torques and the use of a PD feedback structure is derived, where the proportional action is in terms of the vector-quaternion and the two derivative actions are in terms of the airframe angular velocity and the vector-quaternion velocity.

Moreover, in [5] a quaternion-based sliding mode surface is proposed for a model-free of the full dynamic model of a quadrotor. The control algorithm has three important features: the controller assures exponential stability of the full position/attitude dynamics of the system with smooth control efforts, the closed-loop system is robust in presence of external forces and induced moments generated during the flight maneuvers and the controlled quadrotor offers capabilities for aggressive maneuvers. Simulations showed the capabilities of the closed-loop performance under several conditions. Also, [6] proposes a non-linear Proportional squared $\left(P^{2}\right)$ control algorithm fully implemented in the quaternion space, for solving the attitude problem of a quadrotor, the designed control strategy performs very well with a small overshoot and a good reference tracking. However only numerical simulations were presented to prove the efficiency of the suggested scheme.

Meanwhile, [7] introduces the design and experimental flight tests of a quaternion state feedback control scheme to globally stabilize a quadrotor. First an attitude control strategy was proposed to stabilize the vehicle's heading, then a position control law is designed to stabilize the vehicle in all its states. In [8] a comparison between Euler and quaternion approach has been driven, highlighting the efficiency of the second method from a computational point of view. The advantage in considering the quaternion reference is twofold because it avoids critical positions and, it offers a model with the linearity of the coefficients of the transformation matrix, it is also numerically more efficient and stable compared to traditional rotation formulation. Also, in [9] the maximum rate attitude control problem under the input saturation is presented. Moreover, a Backstepping based Inverse Optimal Attitude Controller (BIOAC) is derived which has the property of a maximum convergence rate within the mean- ing of a control Lyapunov function under input torque limitation. In the controller, a backstepping technique is used for handling the complexity introduced by the unit quaternion representation of the attitude of a quadrotor with four parameters.

Besides, there exist a small number of publications, where an energy-based control is designed for an UAV. For example in [10] the physical singularities due to under-actuation are solved by using an energy-based control. Energy-based control is used to overcome the lack of controllability of the quadrotor at physical singular configurations, for instance, when commanding the quadrotor to gain altitude while pitched at $90^{\circ}$. Also, in [11] a cascaded non-linear state feedback control law for a quadrotor is presented, which achieves asymptotic tracking of a predefined position and heading reference trajectory. By a suitable shaping of the potential energy and the injection of a sophisticated damping, this approach enables us to design an outerloop position controller, which satisfies constraints on the maximal and minimal thrust force.

Also, [12] presents a passivity-based approach to stabilize the quadrotor vehicle. However, the authors reduce the problem to the planar maneuvers case to avoid solving complicated Partial Differential Equations (PDEs). Similarly, in [13], a nonlinear control technique based on passivity to solve the path tracking problem for the quadrotor is presented, but only one control loop was considered in this work. The authors showed that the PBC formulation leads to a set of partial differential equations constraints due to the under-actuation degree of the system.

Moreover, [14] introduces a strategy based on a combination of an energy-based and optimal control approaches to stabilize a quadrotor. The system is linearized for solving the well-known Algebraic Riccati Equation (ARE). Simulations have shown that the performance of the proposed control design is satisfactory also in presence of a wind gust perturbation. In [15] a formal method to design a digital inertial control system for quadrotor aircraft is introduced. In particular, it formalizes how to use approximate passive models in order to justify the initial design of energy-based PD controllers.

Therefore in this paper, we propose two control laws to stabilize a quadrotor using energy-based approaches with unit quaternions. The mathematical model based on Euler-Lagrange formulation is written using a quaternion logarithmic mapping. The dynamical model is such 
that an under-actuated system as a quadrotor can be represented as a fully actuated virtual system. The obtained algorithms are based on an energy function and a desired quaternion trajectory. This allows to control the full dynamics of the vehicle. The presented strategies have no singularity problems and were validated in real-time. Furthermore, external disturbances were added to the experiments, showing an effective compensation while flying.

The paper is structured as follows: a brief background of the main concepts and mathematical expressions used in unit quaternions are presented in section II. The quadrotor dynamic model in terms of unit quaternions with the logarithmic mapping is described in section III. The energy-based control strategies are developed in section IV. Numerical simulations to validate the performance of the proposed control strategies are introduced in the section V. Real-time experiments are described in section VI to demonstrate the performance of our proposals in a real system. Finally, conclusions and future work are discussed in section VII.

\section{Quaternion Background}

Quaternions are numbers that can be represented as a sum of a scalar component along an imaginary vector. Let $\boldsymbol{q}$ be a quaternion given by

$\boldsymbol{q}=q_{0}+\bar{q}, \quad q_{0} \in \mathrm{R}, \bar{q} \in \mathrm{R}^{3}$

where $\bar{q}$ denotes the complex vectorial part of $\boldsymbol{q}$, and $q_{0}$ represents the scalar part of $\boldsymbol{q}$.

Quaternions have several operations such as the product, which is defined by

$\boldsymbol{q} \otimes \boldsymbol{r}=\left(q_{0} r_{0}-\bar{q} \cdot \bar{r}\right)+\left(r_{0} \bar{q}+q_{0} \bar{r}+\bar{q} \times \bar{r}\right)$

where $\boldsymbol{r}$ is also a quaternion. The quaternion conjugate can be expressed as $\boldsymbol{q}^{*}=q_{0}-\bar{q}$, the norm by $\|\boldsymbol{q}\|=\sqrt{\boldsymbol{q} \otimes \boldsymbol{q}^{*}}=\sqrt{q_{0}^{2}+q_{1}^{2}+q_{2}^{2}+q_{3}^{2}}$, when $\|\boldsymbol{q}\|=1$, then $\boldsymbol{q}$ is called a unit quaternion. The inverse operator is denoted by $\boldsymbol{q}^{-1}=\|\boldsymbol{q}\|^{-1} \boldsymbol{q}^{*}$. If $\boldsymbol{q}$ is unitary, the inverse and the norm are equivalent.

A unit quaternion can be used to represent the rotation of a rigid body (see Figure 1) using the axis-angle representation and the logarithmic mapping

$\bar{\theta}=2 \ln \boldsymbol{q}, \quad \dot{\bar{\theta}}=\omega$,

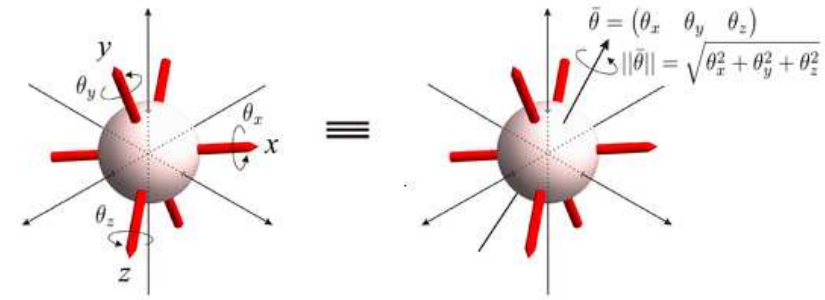

Fig. 1 Axis-angle representation of a rigid body rotation

with

$\ln \boldsymbol{q}= \begin{cases}\ln \|\boldsymbol{q}\|+\frac{\bar{q}}{\|\bar{q}\|} \arccos \frac{q_{0}}{\|\boldsymbol{q}\|}, & \|\bar{q}\| \neq 0 \\ \ln \|\boldsymbol{q}\|, & \|\bar{q}\|=0\end{cases}$

Any vector in a $3 \mathrm{D}$ space can be rotated from one reference frame (say the inertial frame) to another (for example a body frame) using the expression

$v^{\prime}=\boldsymbol{q}^{*} \otimes v \otimes \boldsymbol{q}$

where $v \in \mathbb{R}^{3}$ and $v^{\prime} \in \mathbb{R}^{3}$ are in the inertial and body frames respectively.

The derivate of a quaternion which represents the attitude of a rigid body can be expressed in terms of its orientation and its angular velocity as

$\dot{\boldsymbol{q}}=\frac{1}{2} \boldsymbol{q} \otimes \omega$

\section{Quaternion Mathematical Model}

The quadrotor is an under-actuated system with six degrees of freedom and only four control inputs. Figure 2 shows the vehicle scheme.

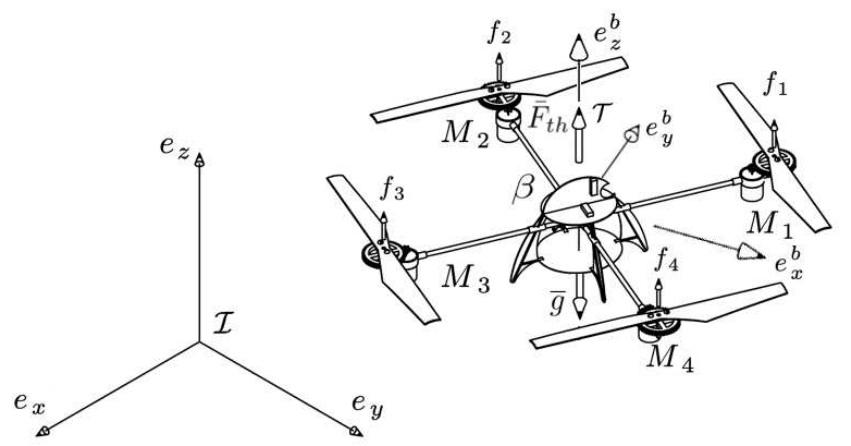

Fig. 2 Quadrotor scheme.

Let us consider an earth fixed frame $I=\left\{e_{x}, e_{y}, e_{z}\right\}$ and body fixed frame $\beta=\left\{e_{x}^{b}, e_{y}^{b}, e_{z}^{b}\right\}$, as seen in Figure 2. $\xi=[p \bar{\theta}]^{T} \in \mathbb{R}^{6}$ denotes all the states variables of 


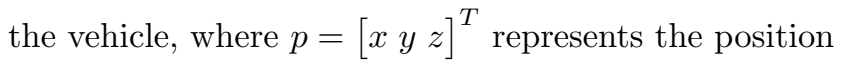
vector with respect to the earth fixed frame, $\bar{\theta}=2 \ln (\boldsymbol{q})$ expresses the rotation quaternion in its axis-angle notation. The vector $\bar{F}_{t h}=\left[\begin{array}{lll}0 & 0 & F_{t h}\end{array}\right]^{T}$ denotes the thrust vector, $\bar{g}=\left[\begin{array}{lll}0 & 0 & -g\end{array}\right]^{T}$ defines the gravity vector, the vector $\tau=\left[\begin{array}{lll}\tau_{u_{x}} & \tau_{u_{y}} & \tau_{u_{z}}\end{array}\right]^{T}$ expresses the torques applied to the body's center of mass, represented on the quadrotor in the body fixed frame.

\subsection{Euler-Lagrange Formulation}

The vehicle motion equations can be obtained by the Euler-Lagrange formulation:

$\frac{d}{d t}\left(\frac{\partial L}{\partial \dot{\xi}}\right)-\frac{\partial L}{\partial \xi}=U$

where $L$ denotes the Lagrangian of the system and is defined as the difference between the kinetic and potential energy,

$L(\xi, \dot{\xi})=K(\dot{\xi})-V(\xi)$,

$U=\left[\begin{array}{ll}F_{u}^{T} & \tau_{u}^{T}\end{array}\right]^{T}$ defines the input vector, which contains $F_{u}$ that denotes the input force with respect to the earth fixed frame and $\tau_{u}$ that represents the input torques expressed in the body fixed frame.

From $(7), K(\dot{\xi})$ expresses the total kinetic energy, which is obtained as follows

$K(\dot{\xi})=\frac{1}{2} m \dot{p}^{T} \dot{p}+\frac{1}{2} \dot{\bar{\theta}}^{T} J \dot{\bar{\theta}}$

and $V(\xi)$ is the total potential energy of the vehicle

$V(\xi)=m g z$

where $m$ represents the quadrotor's mass, $J$ denotes the inertia matrix, $g$ is the gravity, and $z$ describes the vertical position of the vehicle.

Introducing (8) and (9) into (7) we can obtain the Lagrangian equation as follows

$L=\frac{1}{2} m \dot{p}^{T} \dot{p}+\frac{1}{2} \dot{\bar{\theta}}^{T} J \dot{\bar{\theta}}-m g z$,

Then, substituting (10) into (6) the motion equations can be expressed as

$\left[\begin{array}{c}F_{u} \\ \tau_{u}\end{array}\right]=\left[\begin{array}{c}m \ddot{p}-m \bar{g} \\ J \ddot{\bar{\theta}}\end{array}\right]$

where $\ddot{\bar{\theta}}=\tau-\omega \times J \omega$.
Remember that the quadrotor is an under-actuated system, then the force $F_{u}$ which is expressed in the earth fixed frame, is the force $\bar{F}_{t h}$ rotated, see (4), therefore

$F_{u}=\boldsymbol{q} \otimes \frac{\bar{F}_{t h}}{m} \otimes \boldsymbol{q}^{*}$

Since the angular acceleration is given by the external torques and the internal rotational dynamics, it can be expressed as

$\ddot{\bar{\theta}}=\tau-\omega \times J \omega \Rightarrow \tau_{u}=J(\tau-\omega \times J \omega)$.

Besides, the dynamic model (11) can be expressed in matrix form as

$M \ddot{\xi}+G=B U$,

where $M \in \mathbb{R}^{6 \times 6}$ represents the inertia matrix which is symmetric and positive definite, $G \in \mathbb{R}^{6 \times 1}$ defines the gravitational vector and finally, $B \in \mathbb{R}^{6 \times 6}$ is the identity matrix. These matrices are expressed as follows

$M=\left[\begin{array}{cc}m I_{3 \times 3} & 0_{3 \times 3} \\ 0_{3 \times 3} & I_{p} I_{3 \times 3}\end{array}\right]$

$G=\left[\begin{array}{llllll}0 & 0 & m g & 0 & 0 & 0\end{array}\right]^{T}$

where $I_{p}$ denotes the mass moments of the inertia of the vehicle.

Note from (12) that the force in the inertial frame depends on the orientation given by $\boldsymbol{q}$, which varies according to the input torque $\tau$ as seen in (13).

\section{Quaternion-Energy-Based Control Laws}

In this section, the synthesis of quaternion-energy-based controllers for the vehicle are developed.

First, the total energy of the vehicle is obtained and can be described by

$H(\xi, \dot{\xi})=\frac{1}{2} \dot{\xi}^{T} M \dot{\xi}+V(\xi)$

In term of the error function, the total energy is described as

$\bar{H}(\bar{\xi}, \dot{\bar{\xi}})=\frac{1}{2} \dot{\bar{\xi}}^{T} M \dot{\bar{\xi}}+V(\bar{\xi})$

with $\bar{\xi}=\xi-\xi_{d}, \dot{\bar{\xi}}=\dot{\xi}-\dot{\xi_{d}}$, where $\xi_{d}$ represents the desired state vector. 
Now, introducing (9), it yields

$\bar{H}(\bar{\xi}, \dot{\bar{\xi}})=\frac{1}{2} \dot{\bar{\xi}}^{T} M \dot{\bar{\xi}}+m g \bar{z}$

Differentiating the above along the trajectories of the system

$\dot{\bar{H}}=\dot{\bar{\xi}}^{T} M \ddot{\bar{\xi}}+m g \dot{\bar{z}}$

From (16), it follows that

$\dot{\bar{H}}=\dot{\bar{\xi}}^{T} M \ddot{\ddot{\xi}}+\dot{\bar{\xi}}^{T} G$

Substituting (14) into the above, it follows

$\dot{\bar{H}}=\dot{\bar{\xi}}^{T} B U$

Two energy-based control schemes are considered in the following subsections.

\subsection{Case 1}

Now, consider the following positive function as a Lyapunov candidate function

$V(\bar{\xi}, \dot{\bar{\xi}})=\frac{1}{2} K_{E} \bar{H}^{2}+\frac{1}{2} \dot{\bar{\xi}}^{T} K_{m} \dot{\bar{\xi}}+\frac{1}{2} \bar{\xi}^{T} K_{p} \bar{\xi}$

where $K_{p}=K_{p}^{T}>0, K_{m}=K_{m}^{T}>0$ and $K_{E}$ define strictly positive definite constants. Differentiating (21) with respect to time

$\dot{V}(\bar{\xi}, \dot{\bar{\xi}})=K_{E} \bar{H} \dot{\bar{H}}+\dot{\bar{\xi}}^{T} K_{m} \ddot{\bar{\xi}}+\dot{\bar{\xi}}^{T} K_{p} \bar{\xi}$

Introducing (20), we obtain

$\dot{V}(\bar{\xi}, \dot{\bar{\xi}})=K_{E} \bar{H}^{T} B U+\dot{\bar{\xi}}^{T} K_{m} \ddot{\bar{\xi}}+\dot{\bar{\xi}}^{T} K_{p} \bar{\xi}$

Notice from (14) that $\ddot{\bar{\xi}}=M^{-1}(B U-G)$, then

$\dot{V}=K_{E} \bar{H}_{\dot{\xi}^{T}}^{\dot{\xi}^{T}} B U+\dot{\bar{\xi}}^{T} K_{m}\left(M^{-1}(B U-G)\right)+\dot{\bar{\xi}}^{T} K_{p} \bar{\xi}$

Factoring terms, it follows that

$$
\begin{aligned}
\dot{V} & =\dot{\bar{\xi}}^{T}\left(K_{E} \bar{H} B U+K_{m}\left(M^{-1} B U-M^{-1} G\right)+K_{p} \bar{\xi}\right) \\
& =\dot{\bar{\xi}}^{T}\left(\left[K_{E} \bar{H} B+K_{m} M^{-1} B\right] U-K_{m} M^{-1} G+K_{p} \bar{\xi}\right)
\end{aligned}
$$

Therefore, the first control law is defined such that:

$$
\left[K_{E} \bar{H} B+K_{m} M^{-1} B\right] U-K_{m} M^{-1} G+K_{p} \bar{\xi}=-K_{v} \dot{\bar{\xi}}
$$

where $K_{v}=K_{v}^{T}>0$.
This leads to

$\dot{V}(\bar{\xi}, \dot{\bar{\xi}})=-\dot{\bar{\xi}}^{T} K_{v} \dot{\bar{\xi}}$

From (22) we can obtain

$U=[E]^{-1}\left[-K_{p} \bar{\xi}-K_{v} \dot{\bar{\xi}}+K_{m} M^{-1} G\right]$

where $E=K_{E} \bar{H} B+K_{m} M^{-1} B$.

$B$ is an identity matrix, this ensures that $E$ always has inverse and that $U$ does not have singularities.

The final control law can be rewritten, as follows

$\left[\begin{array}{c}F_{u} \\ \tau_{u}\end{array}\right]=[E]^{-1}\left[\begin{array}{c}-K_{p t}\left(p-p_{d}\right)-K_{v t}\left(\dot{p}-\dot{p}_{d}\right)-K_{m t} \bar{g} \\ -2 K_{p r} \ln \left(\boldsymbol{q}_{e}\right)-K_{v r}\left(\dot{\bar{\theta}}-\dot{\bar{\theta}}_{d}\right)\end{array}\right]$

where $K_{p t}>0, K_{p r}>0, K_{v t}>0, K_{v r}>0$ and $K_{m t}>0$ contain design parameters, $p_{d}$ denotes the equilibrium configuration, $\bar{\theta}_{d}=2 \ln \boldsymbol{q}_{d}$. Take into account that $\boldsymbol{q}_{e}=\boldsymbol{q} \otimes \boldsymbol{q}_{d}^{*}$ defines the quaternion error between the actual orientation $\boldsymbol{q}$ and the desired reference $\boldsymbol{q}_{d}^{*}$. If the control law is such that $\ln \left(\boldsymbol{q}_{e}\right) \rightarrow\left[\begin{array}{lll}0 & 0 & 0\end{array}\right]^{T}$, then $\boldsymbol{q}_{e} \rightarrow 1+\left[\begin{array}{lll}0 & 0 & 0\end{array}\right]^{T}$, which implies that the orientation of the vehicle converges to the desired reference $\boldsymbol{q} \rightarrow \boldsymbol{q}_{d}^{*}$

$F_{u}$ expresses the desired force expressed in the inertial frame which will stabilize the quadrotor in the desired position, and $\tau_{u}$ represents the torque that makes the attitude converge to the desired quaternion reference.

The quaternion trajectory $\boldsymbol{q}_{d}$ is defined as follows

$$
\begin{aligned}
\boldsymbol{q}_{d} & =\frac{\left(b \cdot F_{u}+\left\|F_{u}\right\|\right)+b \times F_{u}}{\left\|\left(b \cdot F_{u}+\left\|F_{u}\right\|\right)+b \times F_{u}\right\|}, \\
F_{t h} & =\left\|F_{u}\right\|
\end{aligned}
$$

where $b=\left[\begin{array}{lll}0 & 0 & 1\end{array}\right]^{T}$ denotes the axis on which the thrust acts in the body fixed frame.

From (24) $\boldsymbol{q}_{d}$ is used to close the loop such that $\bar{F}_{t h}$ is rotated to coincide with $F_{u}$, thus the position is stabilized in the desired reference. The above is justified by well known time-scale separation between rotational and translational dynamics. Thus, the control law (23) in quaternion space guarantees the stabilization of all the system states. 


\subsection{Case 2}

In this case an energy-based optimal control law using unit-quaternions is proposed.

Considering the state vector as $x(t)=[\bar{\xi} \dot{\bar{\xi}}]^{T}=$ $[p \bar{\theta} \dot{p} \dot{\bar{\theta}}]^{T}$. Then, system (11) can be rewritten as follows:

$$
\left[\begin{array}{c}
\dot{x}_{1} \\
\dot{x}_{2} \\
\dot{x}_{3} \\
\dot{x}_{4}
\end{array}\right]=\left[\begin{array}{c}
x_{2} \\
x_{4} \\
-g \\
0
\end{array}\right]+\left[\begin{array}{c}
0 \\
0 \\
m^{-1} F_{u} \\
J^{-1} \tau_{u}
\end{array}\right]
$$

Now, the performance cost function which is to be minimized is defined as follows:

$J=\frac{1}{2} \int_{0}^{\infty}\left(x^{T} Q x+u^{T} R u\right) d t$

where the state and input weighting matrices are assumed such that $Q=Q^{T}, Q>0$ and $R=R^{T}, R \geqslant 0$.

System (25) can be optimally stabilized solving:

$\frac{d V_{o}}{d t}+x^{T} Q x+u^{T} R u=0$

Then, consider the the following Lyapunov candidate function based on the total energy

$V_{o}=\frac{1}{2} K_{E} \bar{H}^{2}+\frac{1}{2} \dot{\bar{\xi}}^{T} K_{m} \dot{\bar{\xi}}+\frac{1}{2} \bar{\xi}^{T} K_{p} \bar{\xi}+\bar{\xi}^{T} K_{T} \dot{\bar{\xi}}$

where $K_{T}=K_{T}{ }^{T}>0$. Differentiating (28) along the trajectories of the system

$\dot{V}_{o}(\bar{\xi}, \dot{\bar{\xi}})=K_{E} \bar{H} \dot{\bar{H}}+\dot{\bar{\xi}}^{T} K_{m} \ddot{\bar{\xi}}+\dot{\bar{\xi}}^{T} K_{p} \bar{\xi}+\bar{\xi}^{T} K_{T} \ddot{\bar{\xi}}+\dot{\bar{\xi}}^{T} K_{T} \dot{\bar{\xi}}$

Now, introducing (19) in the above, it follows that

$$
\begin{aligned}
\dot{V}_{o}= & K_{E} \bar{H}\left(\dot{\bar{\xi}} M \ddot{\bar{\xi}}+\dot{\bar{\xi}}^{T} G\right)+\dot{\bar{\xi}}^{T} K_{m} \ddot{\bar{\xi}}+\dot{\bar{\xi}}^{T} K_{p} \bar{\xi}+\dot{\xi}^{T} K_{T} \ddot{\bar{\xi}} \\
& +\dot{\bar{\xi}}^{T} K_{T} \dot{\bar{\xi}} \\
= & \left(K_{E} \bar{H} \dot{\bar{\xi}}^{T} M+\dot{\dot{\xi}}^{T} K_{m}+\bar{\xi}^{T} K_{T}\right) \ddot{\bar{\xi}}+K_{E} \bar{H}^{\dot{\bar{\xi}}^{T}} G \\
& +\dot{\bar{\xi}}^{T} K_{p} \bar{\xi}+\dot{\bar{\xi}}^{T} K_{T} \dot{\bar{\xi}}
\end{aligned}
$$

Substituting (14) into (29), it yields

$$
\begin{aligned}
\dot{V}_{o}= & \left(K_{E} \bar{H} \dot{\bar{\xi}}^{T} M+\dot{\bar{\xi}}^{T} K_{m}+\bar{\xi}^{T} K_{T}\right) M^{-1}(U-G) \\
& +K_{E} \bar{H} \dot{\bar{\xi}}^{T} G+\dot{\bar{\xi}}^{T} K_{p} \bar{\xi}+\dot{\bar{\xi}}^{T} K_{T} \dot{\bar{\xi}}
\end{aligned}
$$

Finally, introducing (30) into (27) and applying dynamic programming, it follows that

$$
\begin{aligned}
0= & \frac{\partial}{\partial(U-G)}\left[+K_{E} \overline{\dot{H}} \dot{\bar{\xi}}^{T} G+\dot{\bar{\xi}}^{T} K_{p} \bar{\xi}+\dot{\bar{\xi}}^{T} K_{T} \dot{\bar{\xi}}+x^{T} Q x\right. \\
& \left(K_{E} \bar{H}_{\bar{\xi}}^{T} M+\dot{\bar{\xi}}^{T} K_{m}+\bar{\xi}^{T} K_{T}\right) M^{-1}(U-G) \\
& \left.+(U-G)^{T} R(U-G)\right]
\end{aligned}
$$

Then,

$$
K_{E} \bar{H} \dot{\bar{\xi}}^{T}+\left(\dot{\bar{\xi}}^{T} K_{m}+\bar{\xi}^{T} K_{T}\right) M^{-1}+R(U-G)=0
$$

Therefore, the control law can be represented as

$U=-R^{-1}\left[K_{E} \bar{H} \dot{\bar{\xi}}+\left(\dot{\bar{\xi}}^{T} K_{m}+\bar{\xi}^{T} K_{T}\right) M^{-1}\right]+G$

The final control law can be rewritten, as follows

$$
\begin{aligned}
{\left[\begin{array}{c}
F_{u} \\
\tau_{u}
\end{array}\right]=} & R^{-1}\left[M^{-1}\left[\begin{array}{l}
-K_{p t}\left(p-p_{d}\right)-K_{v t}\left(\dot{p}-\dot{p}_{d}\right) \\
-2 K_{p r} \ln \left(\boldsymbol{q}_{e}\right)-K_{v r}\left(\dot{\bar{\theta}}-\dot{\bar{\theta}}_{d}\right)
\end{array}\right]\right. \\
& \left.-K_{E} \bar{H}\left[\begin{array}{c}
\left(\dot{p}-\dot{p}_{d}\right) \\
\left(\dot{\bar{\theta}}-\dot{\bar{\theta}}_{d}\right)
\end{array}\right]\right]+\left[\begin{array}{c}
-m \bar{g} \\
0
\end{array}\right]
\end{aligned}
$$

Remember that $\bar{\theta}_{d}=2 \ln \boldsymbol{q}_{d}$ and $q_{e}=\boldsymbol{q} \otimes \boldsymbol{q}_{d}^{*}$.

\section{Numerical Validation}

Our laboratory has developed a simulator which is fully compatible with our drones, and models in a very precise way the dynamics of the UAV, see Figure 3 . Numerical simulations were used to validate both of the proposed control schemes using this simulation environment.

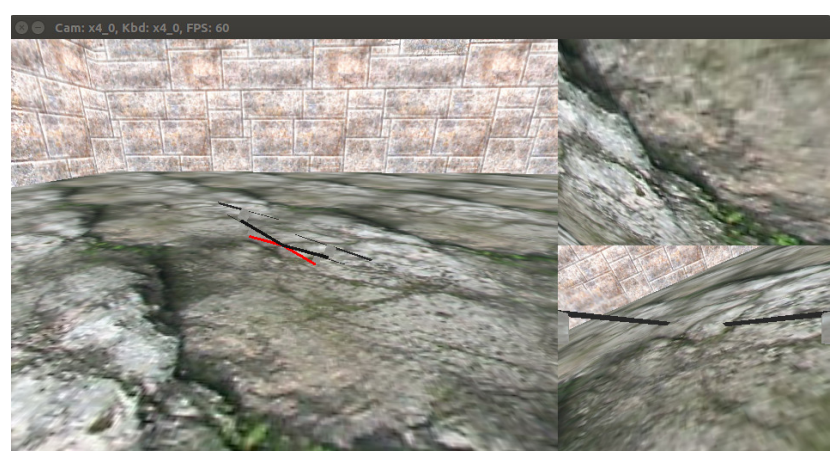

Fig. 3 Simulation environment for a quadrotor vehicle.

Our platform uses an optical tracking system to measure the position the vehicle with an array of cameras. The references are considered to be in the NED (North, East, Down) convention, where the $x$ axis is pointing the front of the drone, the $y$ points its right 
side, and the $z$ axis points down.

The control gains were considered to be diagonal matrices, and were adjusted empirically to obtain a stable behavior of the simulation.

$$
\begin{array}{ll}
K_{p t}=\operatorname{diag}(0.25,0.25,2) & K_{p r}=\operatorname{diag}(6,6,6) \\
K_{v t}=\operatorname{diag}(0.125,0.125,0.5) & K_{v r}=\operatorname{diag}(0.5,0.5,1) \\
K_{m}=\operatorname{diag}\left(m I^{3 \times 3}, J\right) & K_{E}=0.05
\end{array}
$$

The UAV platform was considered geometrically symmetric such that the mass and the inertial matrix can be defined as

$J=\left[\begin{array}{ccc}0.177 & 0 & 0 \\ 0 & 0.177 & 0 \\ 0 & 0 & 0.354\end{array}\right], \quad m=408 g$

\subsection{Simulated Scenario}

A trajectory was computed such that the vehicle follows a circular path in the horizontal plane while maintaining a constant altitude. The reference points were calculated as

$p_{d}=\left[\begin{array}{l}-r \cos \left(t_{c}\right)+r \\ -r \sin \left(t_{c}\right) \\ z_{d}\end{array}\right]$,

where $t_{c}$ represents a discrete time which starts in zero when the trajectory begins and increments in steps of $\Delta t_{c}=0.006$ in each computer cycle, and $z_{d}$ is the desired altitude which is considered to be constant.

For this simulation, a $r=1 \mathrm{~m}$ circle was considered for the first two loops, then the radius was abruptly changed to $r=2 m$ until two more loops are made. In this case, the heading of the quadrotor is kept constant at zero during the circling movement.

The position signals and references were used to compute a desired force (see Figures 4 and 5) to drive the vehicle towards the trajectory.

The values of these graphs express the fraction of the total force the quad-rotor's propellers are able to exert, with $\left\|F_{x}\right\|,\left\|F_{y}\right\|,\left\|F_{z}\right\|<1$, where 1 means the motors are rotating at their maximum speed.

Using (24), an attitude trajectory is computed to make the thrust force coincide with the desired force, these trajectories are illustrated in Figures 6 and 7.

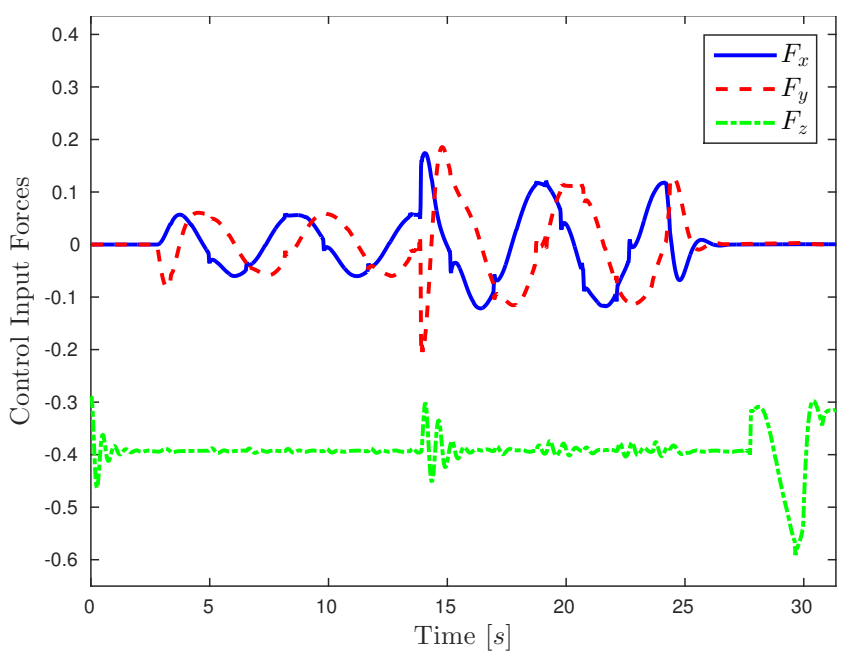

Fig. 4 Input forces for case 1.

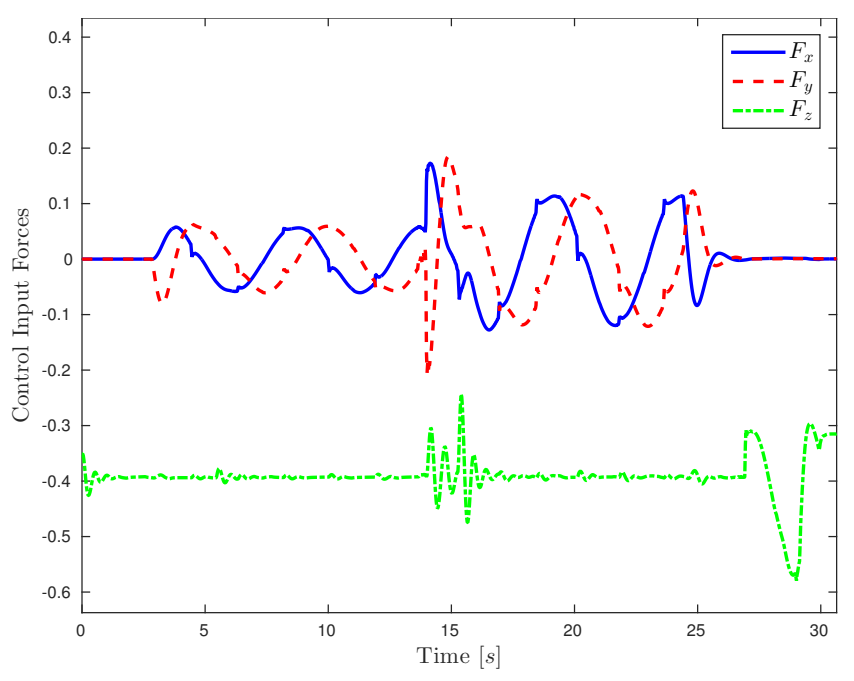

Fig. 5 Input forces for case 2.

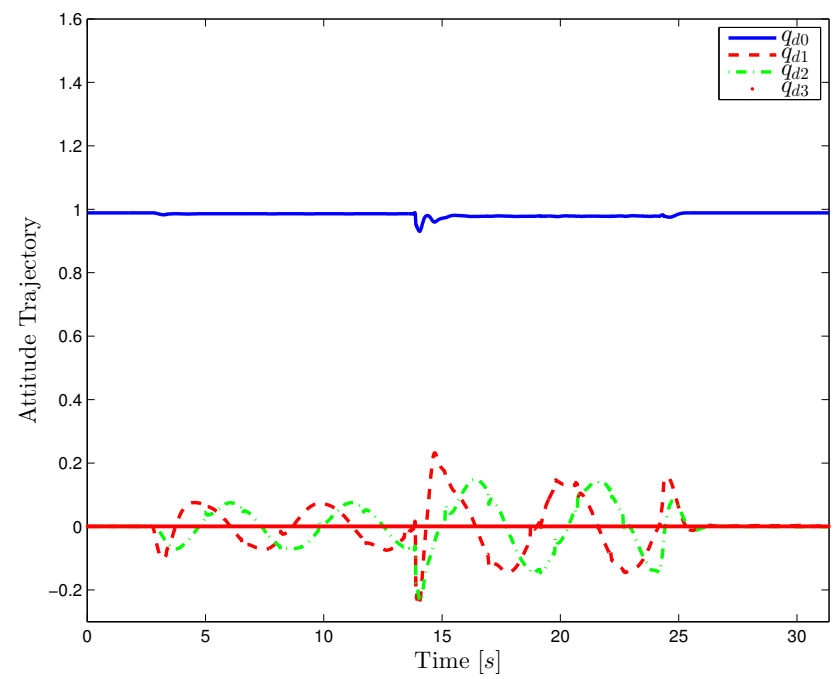

Fig. 6 Quad-rotor attitude reference $\left(q_{d}\right)$ in case 1. 


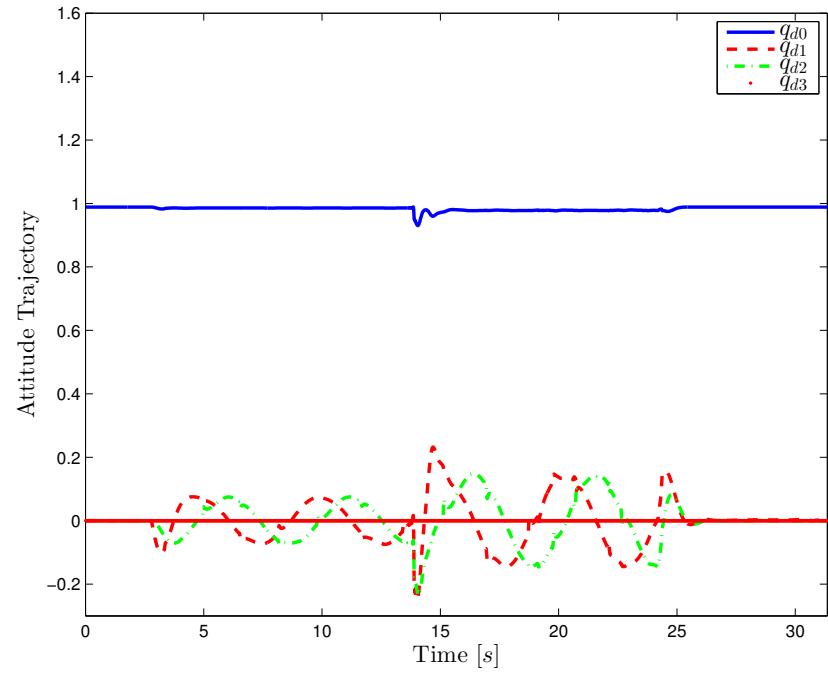

Fig. 7 Quad-rotor attitude reference $\left(q_{d}\right)$ in case 2.

The rotational inputs are calculated such that $\boldsymbol{q}$ follows $\boldsymbol{q}_{d}$. Similarly to the input forces, torques are expressed in values $\left\|\tau_{x}\right\|, \mid \tau_{y}\|,\| \tau_{z} \|<1$, where 0 means no torque and \pm 1 means the maximum moment in either direction.

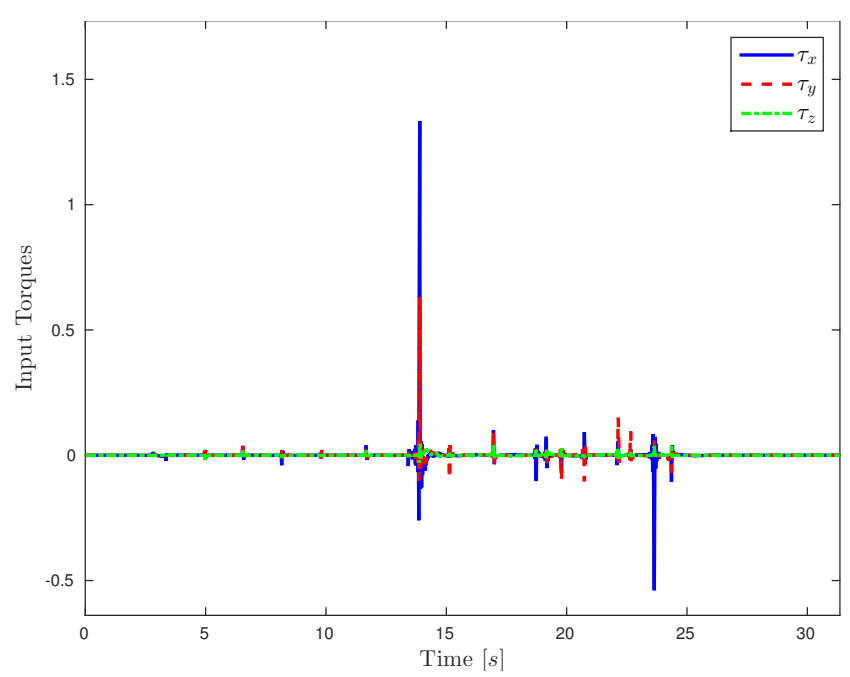

Fig. 8 Input torques for case 1.

In Figures 10 through 15 the vehicle's attitude is illustrated independently for each component of the quaternion. Note the vehicle's attitude follows the computed trajectory in a very precise manner, the $q_{3}$ component axis was not plotted because its rotational dynamics are decoupled from the translation, so its values stay in zero.

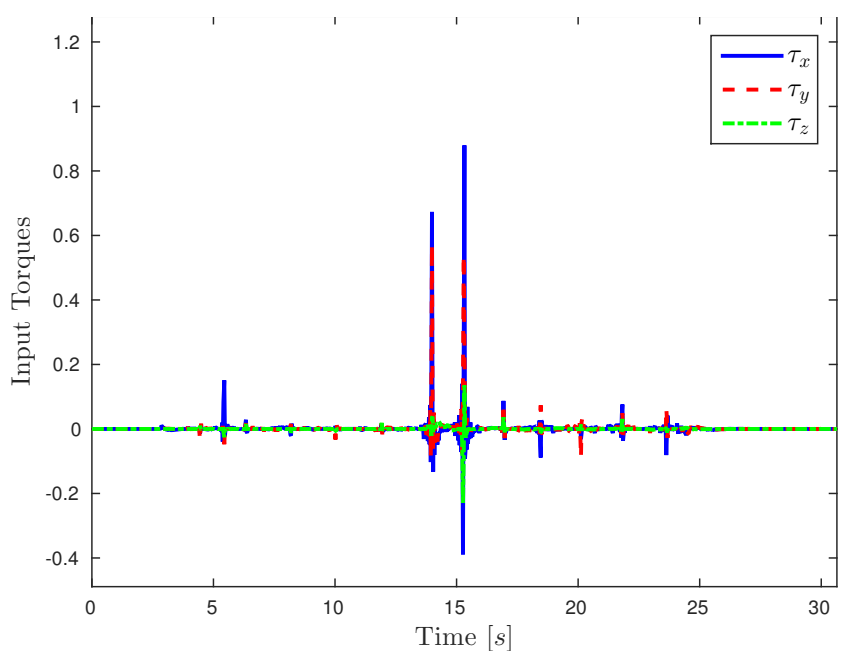

Fig. 9 Input torques for case 2.

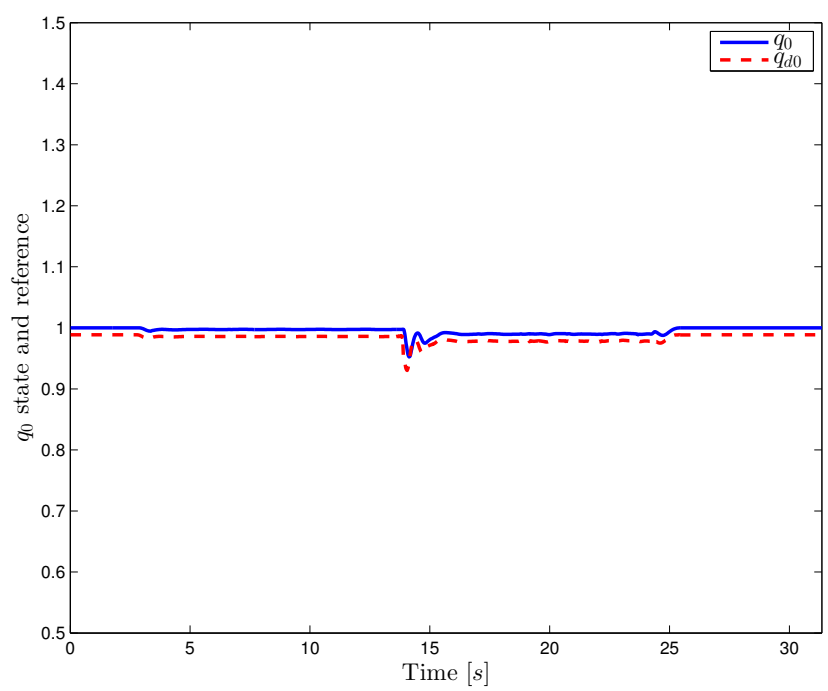

Fig. 10 Attitude and reference for $q_{0}$ in case 1.

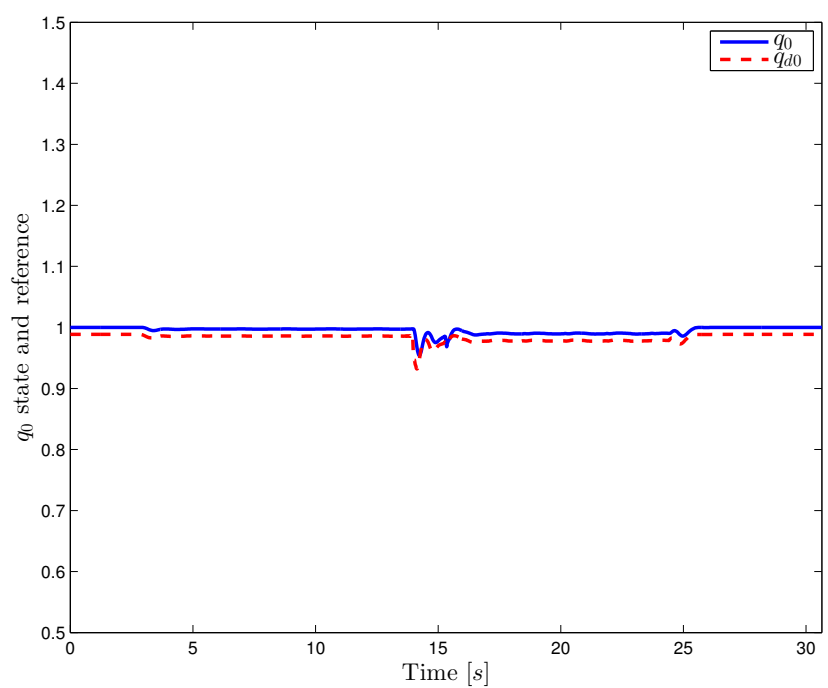

Fig. 11 Attitude and reference for $q_{0}$ in case 2. 


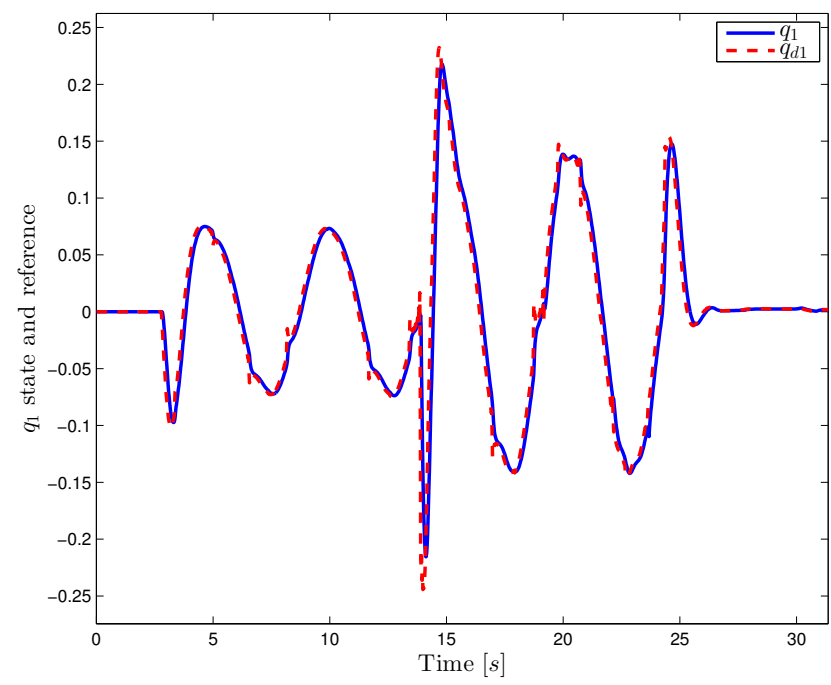

Fig. 12 Attitude and reference for $q_{1}$ in case 1.

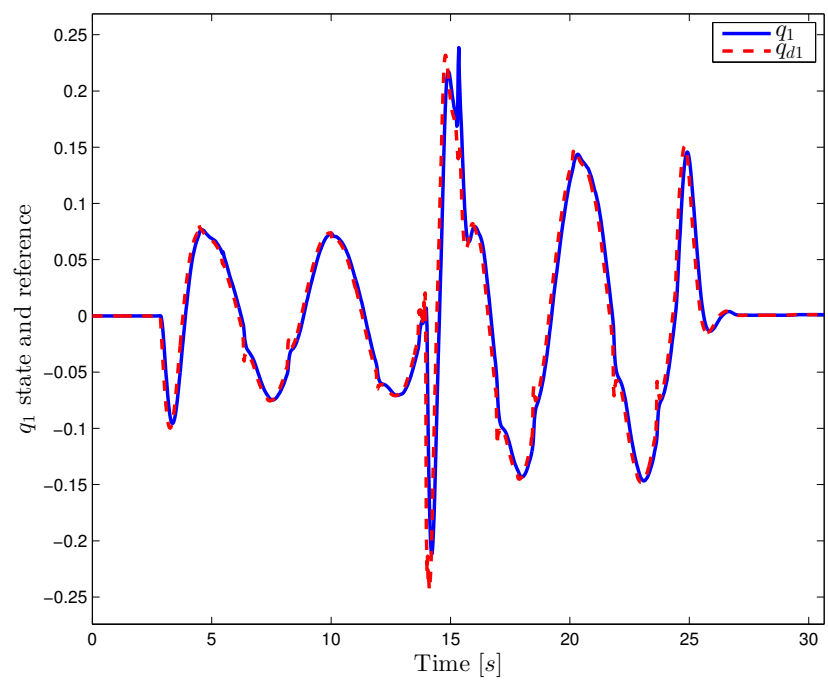

Fig. 13 Attitude and reference for $q_{1}$ in case 2 .

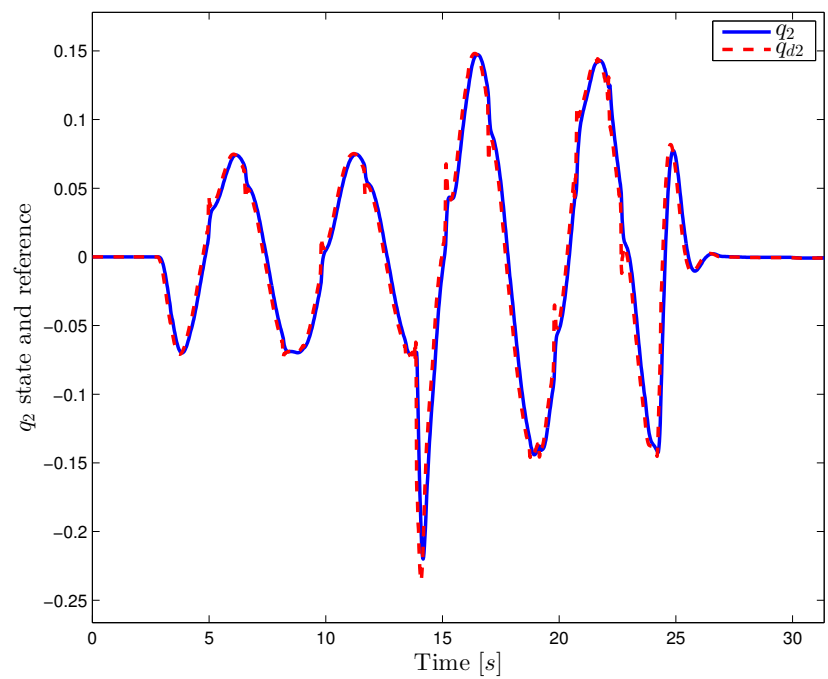

Fig. 14 Attitude and reference for $q_{2}$ in case 1 .

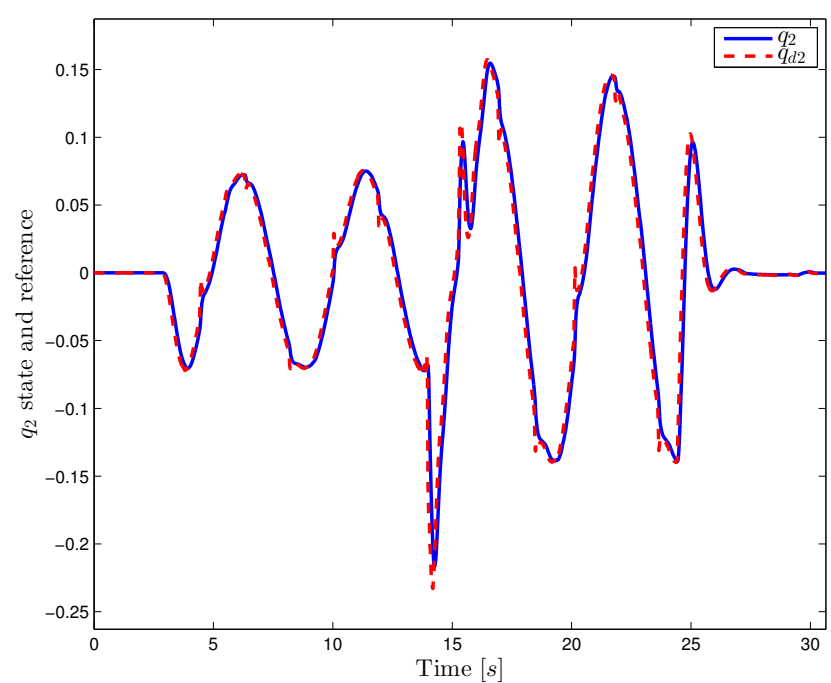

Fig. 15 Attitude and reference for $q_{2}$ in case 2 .

In order to illustrate the attitude behavior in a more comprehensive manner for the reader, a conversion from quaternion to Euler angles was applied as

$$
\begin{gathered}
\phi=\operatorname{atan} 2\left(2\left(q_{0} q_{1}+q_{2} q_{3}\right), 1-2\left(q_{1} q_{1}+q_{2} q_{2}\right)\right) \\
\theta=\operatorname{asin}\left(2\left(q_{0} q_{2}-q_{1} q_{3}\right)\right) \\
\psi=\operatorname{atan} 2\left(2\left(q_{0} q_{3}+q_{1} q_{2}\right), 1-2\left(q_{2} q_{2}+q_{3} q_{3}\right)\right)
\end{gathered},
$$

The roll, pitch, and yaw angles are illustrated in Figures 1621 .

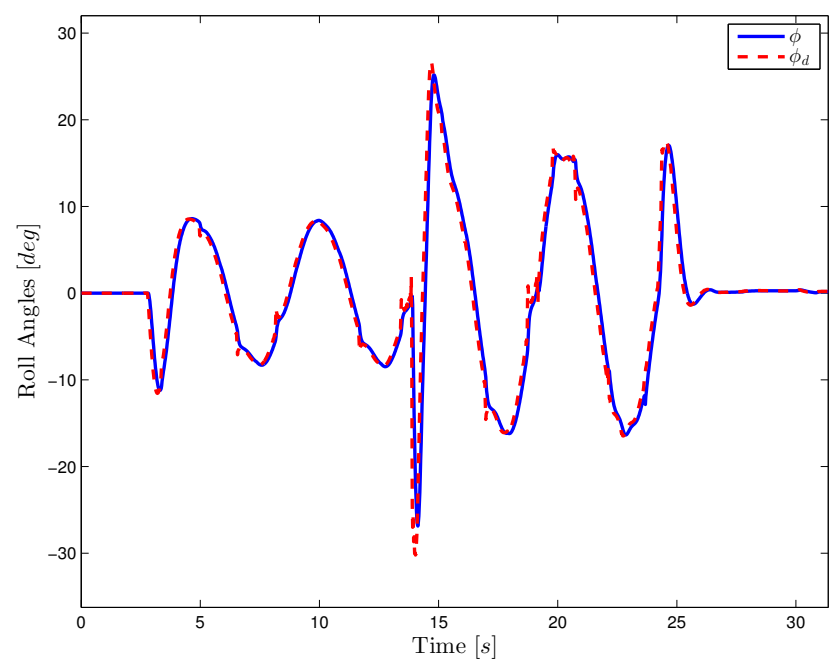

Fig. 16 Attitude and reference for $\phi$ angles in case 1. 


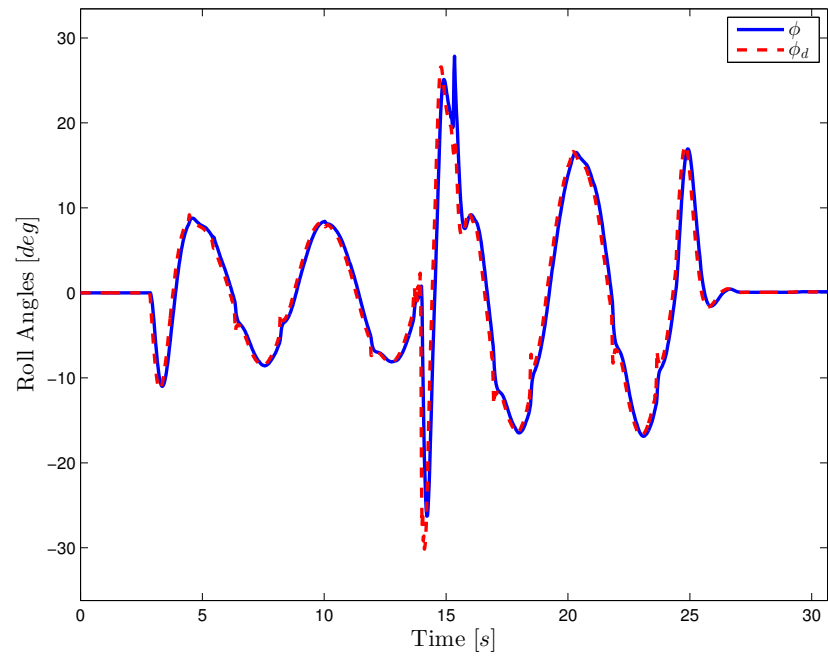

Fig. 17 Attitude and reference for $\phi$ angles in case 2 .

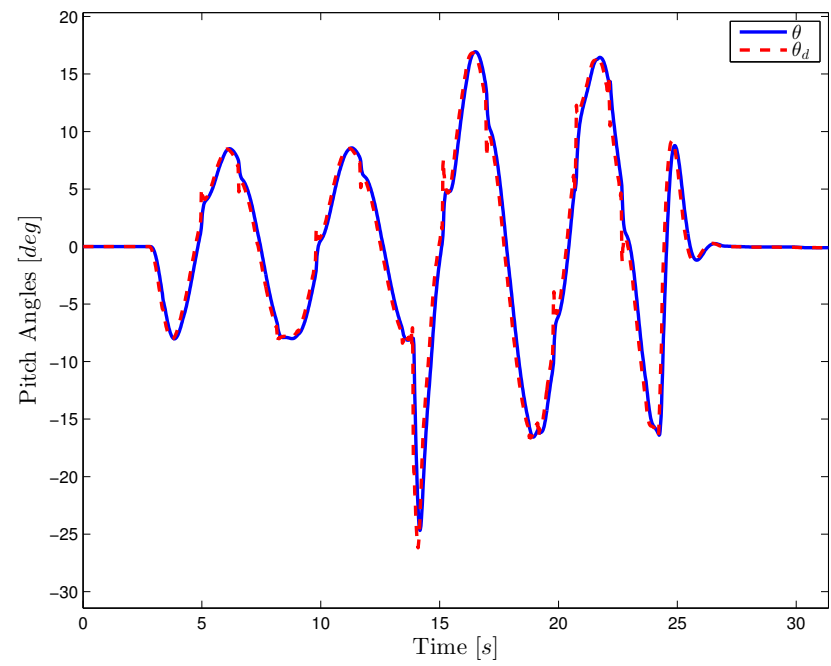

Fig. 18 Attitude and reference for $\theta$ angles in case 1 .

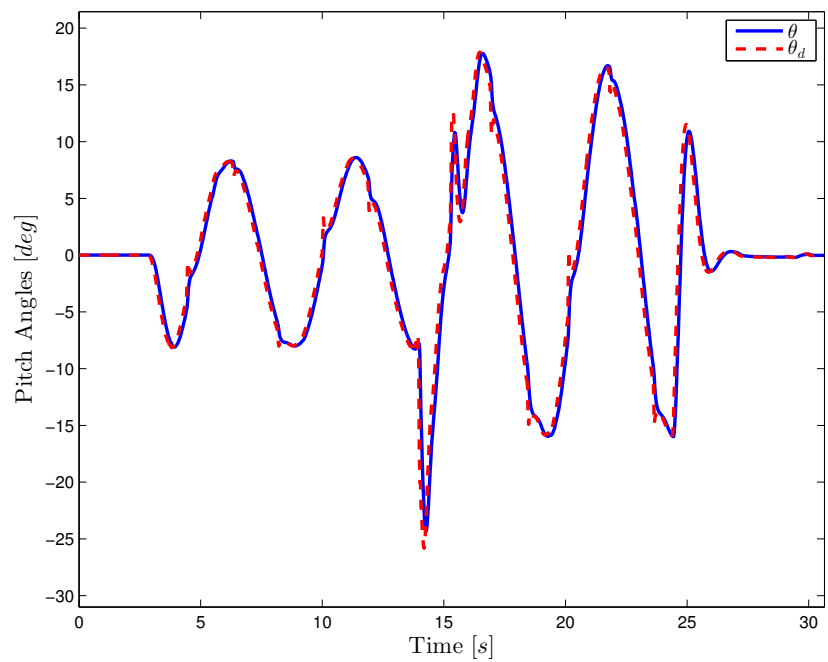

Fig. 19 Attitude and reference for $\theta$ angles in case 2 .

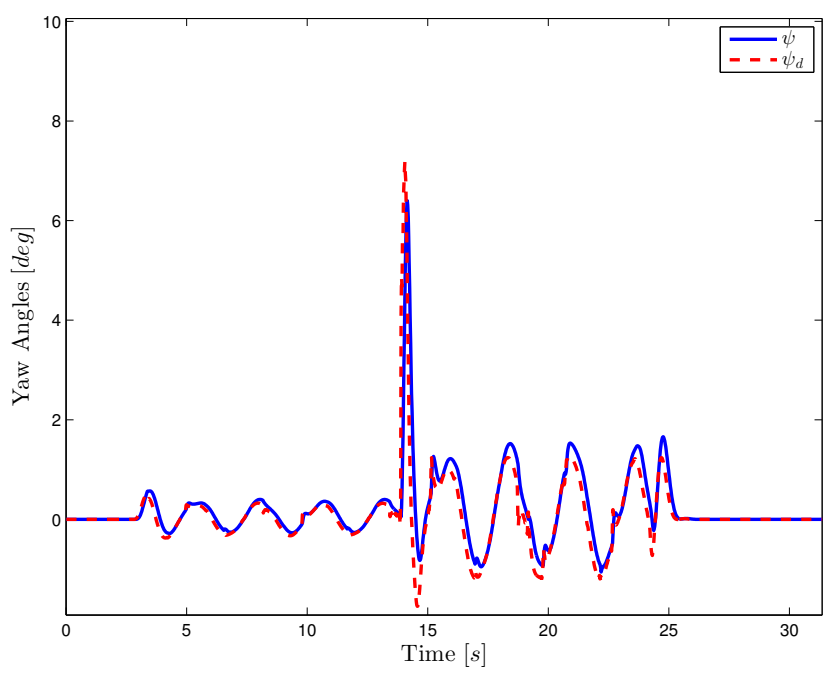

Fig. 20 Attitude and reference for $\psi$ angles in case 1.

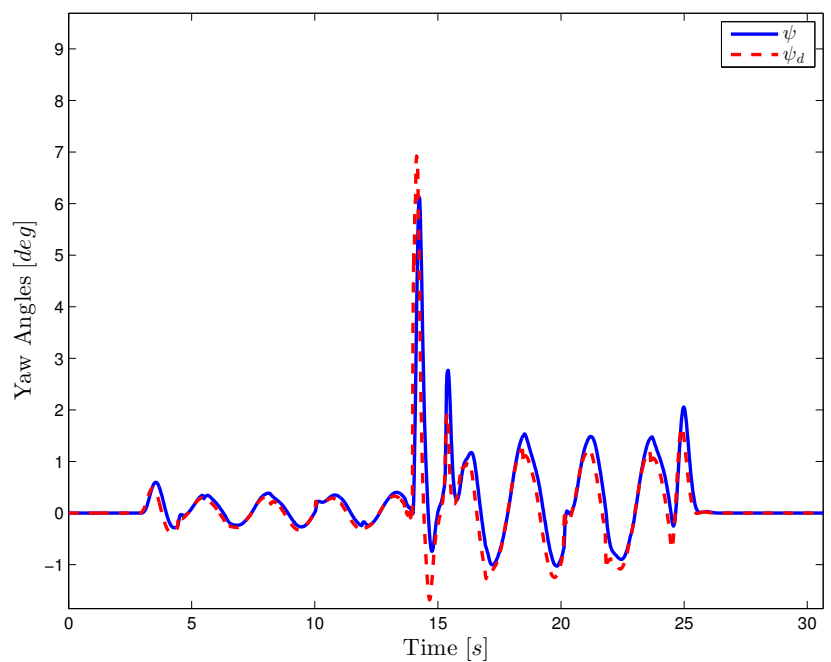

Fig. 21 Attitude and reference for $\psi$ angles in case 2 .

Figures 22 through 27 represent the translational dynamics. Note that the position for each axis is stabilized according to the trajectory.

Lastly, Figures 28 and 29 illustrates the circles described by the quad-rotor in the horizontal plane. 


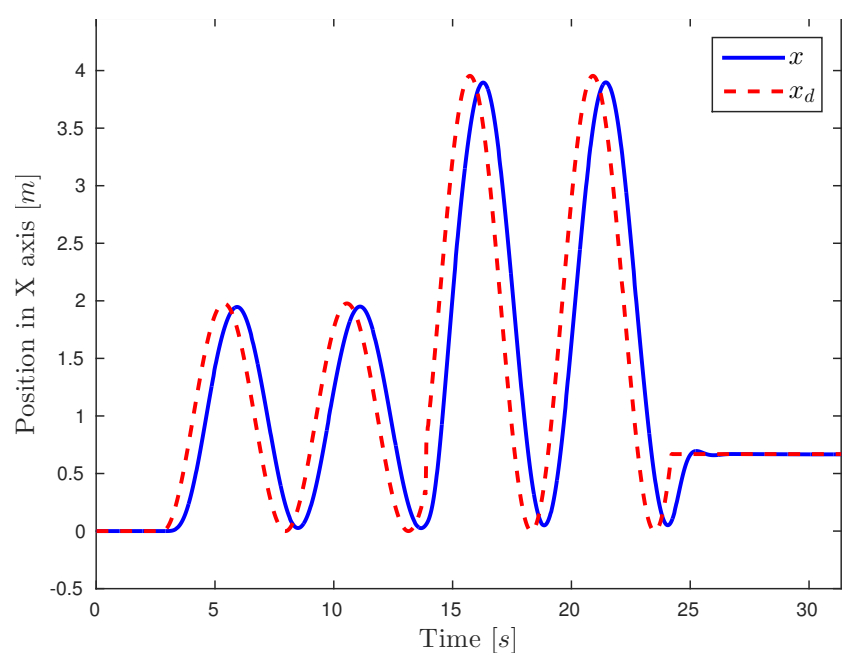

Fig. 22 Quad-rotor's position in the $x$ axis in case 1 .

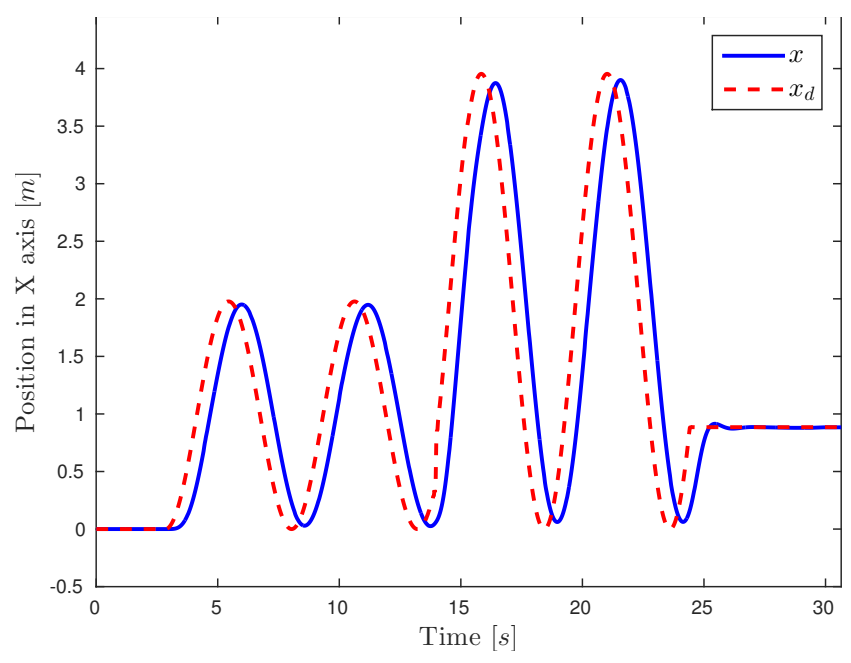

Fig. 23 Quad-rotor's position in the $x$ axis in case 2 .

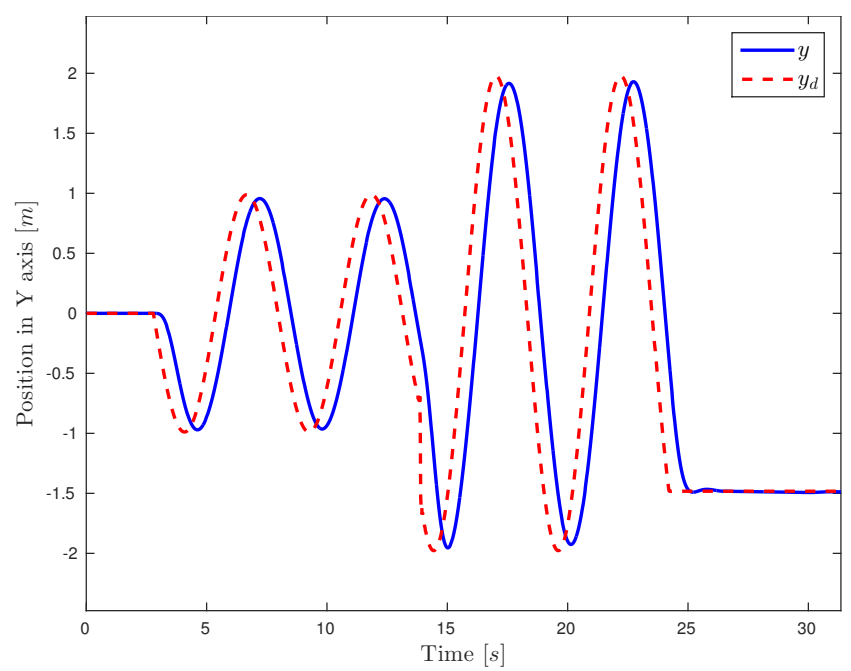

Fig. 24 Quad-rotor's position in the $y$ axis in case 1.

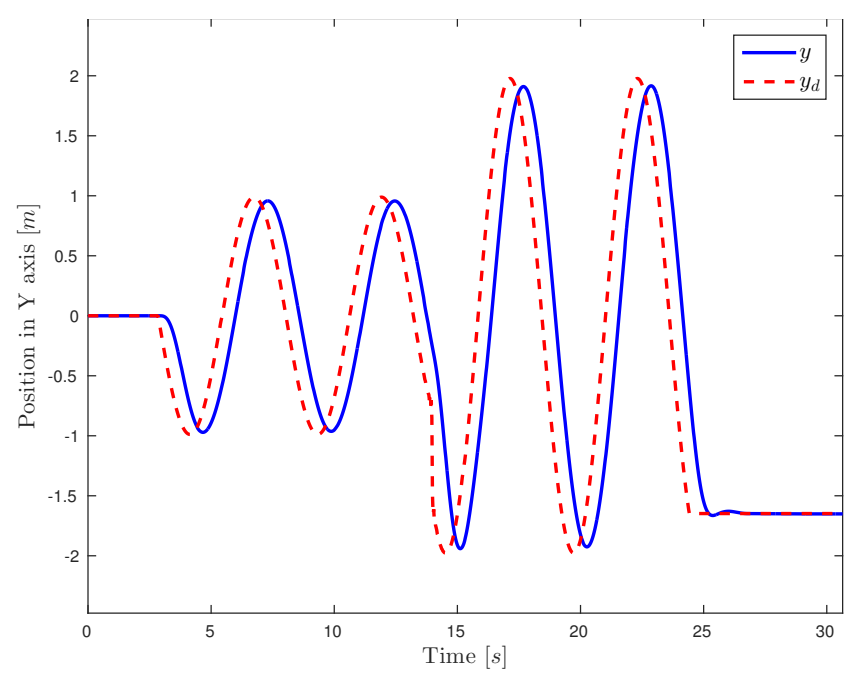

Fig. 25 Quad-rotor's position in the $y$ axis in case 2 .

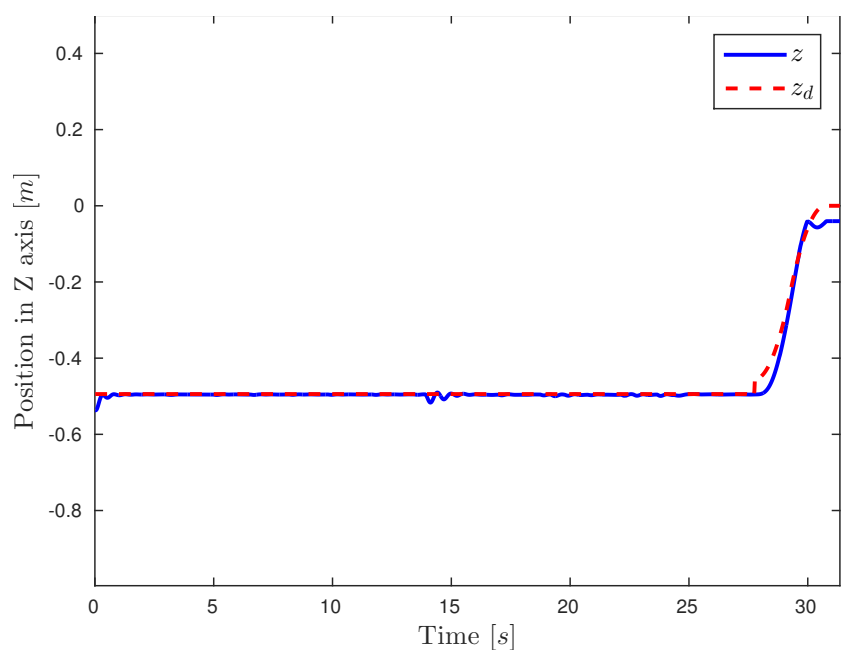

Fig. 26 Quad-rotor's position in the $z$ axis in case 1.

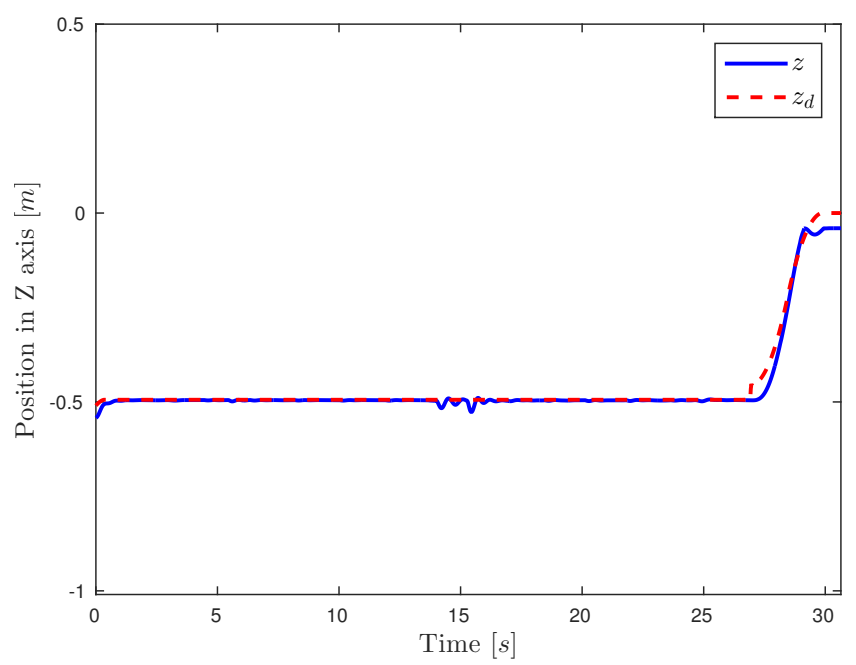

Fig. 27 Quad-rotor's position in the $z$ axis in case 2 . 


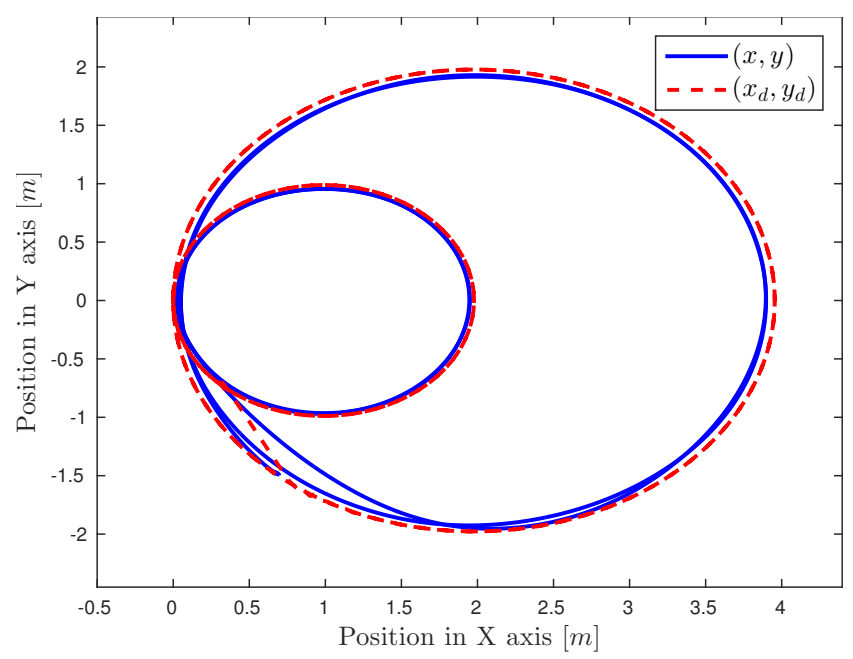

Fig. 28 Quadrotor's horizontal translation in case 1.

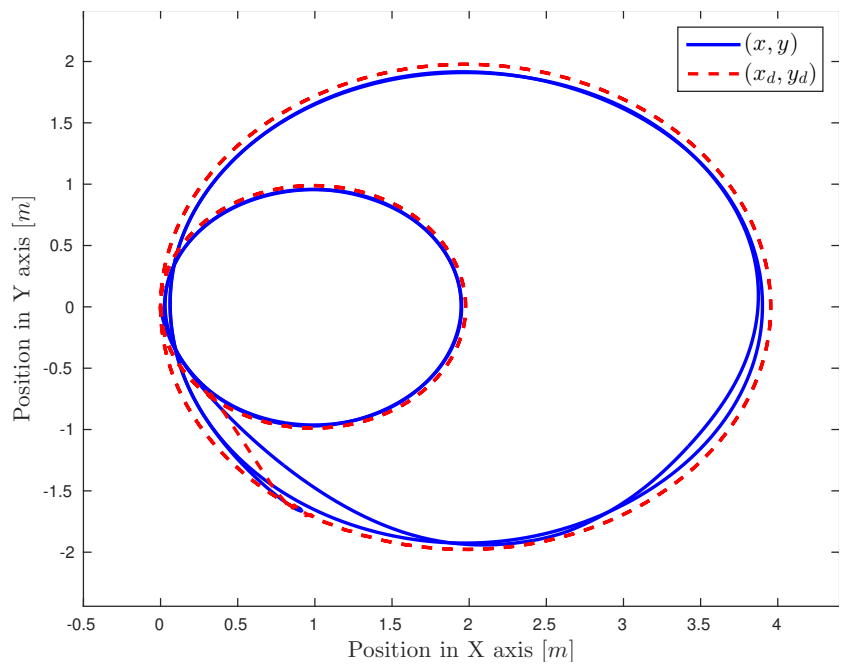

Fig. 29 Quadrotor's horizontal translation in case 2 .

\section{Flight Tests}

The Parrot AR Drone 2 was then used to perform tests in real experiments, this UAV has been adapted to work under our laboratorys framework.

An Inertial Measurement Unit (IMU), and an OptiTrack motion capture system were used to measure the rotational and translational position and velocities.

\subsection{Circular trajectory}

A circular trajectory, similar to the one presented in the simulation, was introduced to the quad-rotor which is illustrated in Figures 30 and 31 for each case.
In this case, the experiments consisted in a first $r=$ $1 \mathrm{~m}$ circle and immediately followed by another one with $r=1.5 \mathrm{~m}$. As in the simulated experiment, the heading of the quadrotor is kept constant at zero during the circling movement.

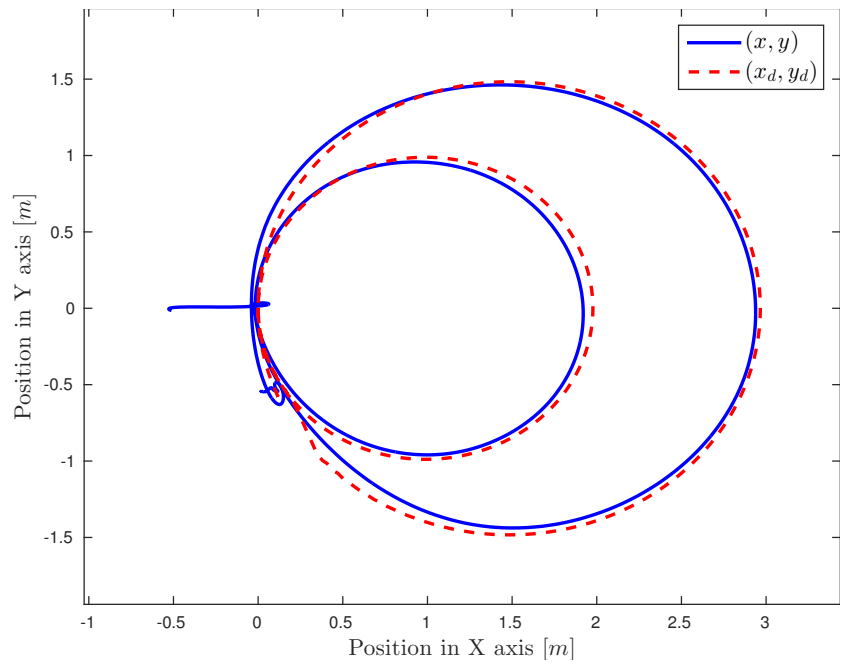

Fig. 30 Quad-rotor's horizontal translation in case 1 experiments.

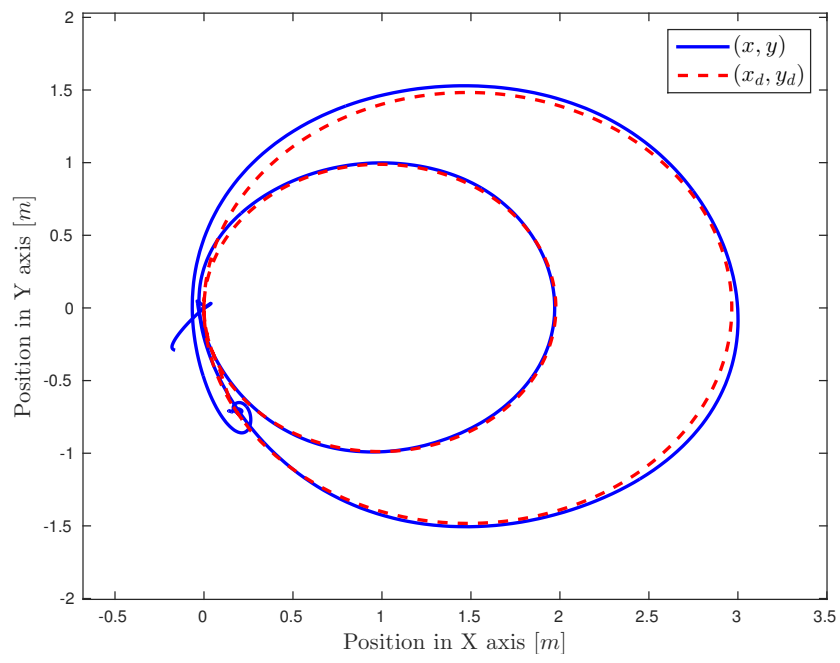

Fig. 31 Quad-rotor's horizontal translation in case 2 experiments.

A control force is computed with the position error, this is represented in Figures 32 and 33. 


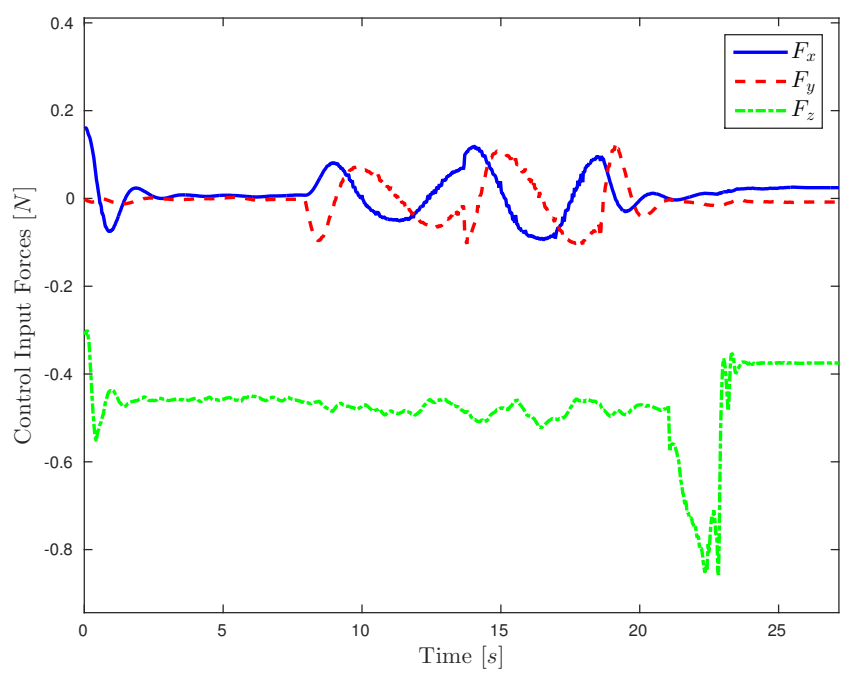

Fig. 32 Quad-rotor's control forces for case 1.

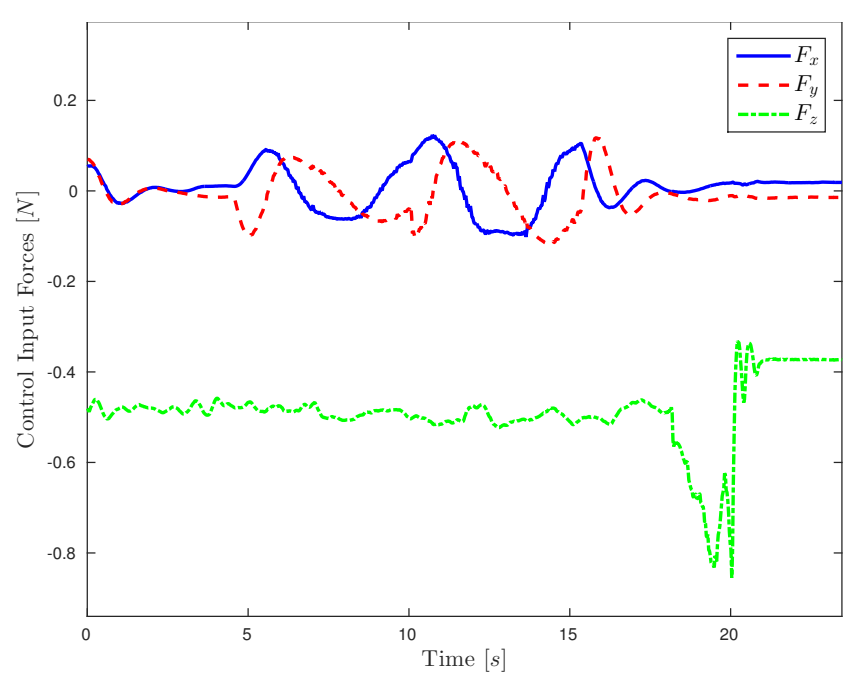

Fig. 33 Quad-rotor's control forces for case 2.

A quaternion attitude trajectory is computed to orient the thrust force to the direction of the control force using the designed controller, this is illustrated in Figures 34 through 39 .

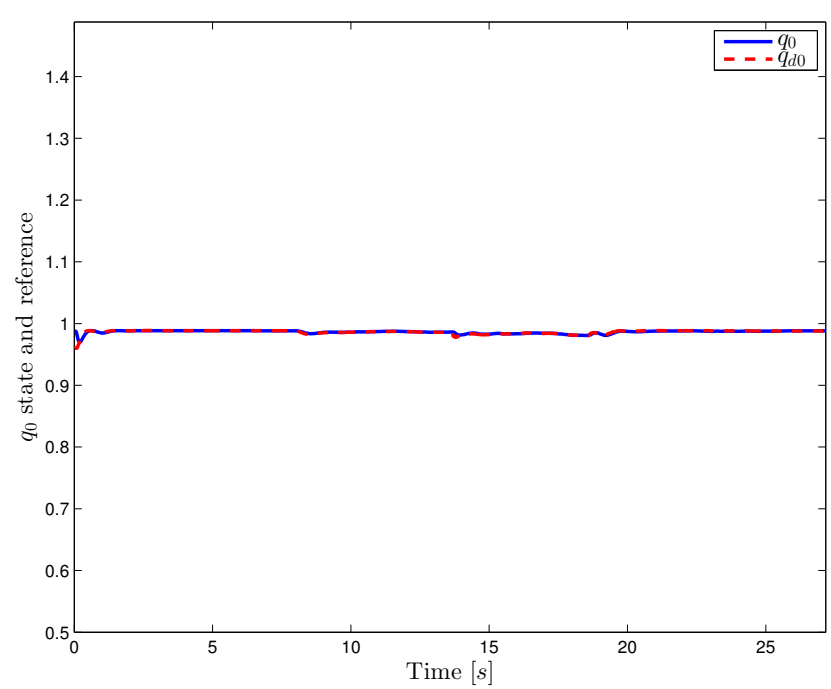

Fig. 34 Attitude and reference for $q_{0}$ in case 1 experiments.

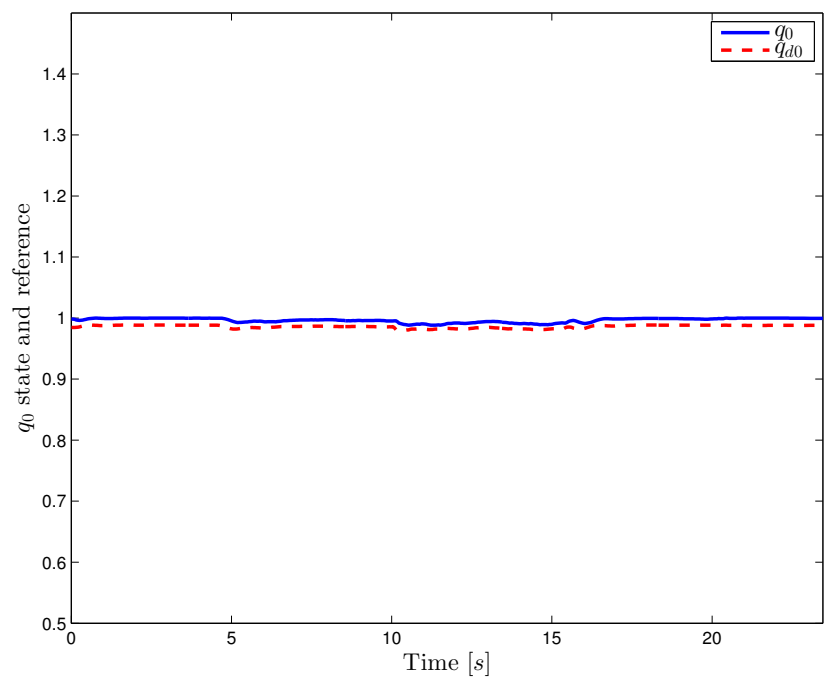

Fig. 35 Attitude and reference for $q_{0}$ in case 2 experiments.

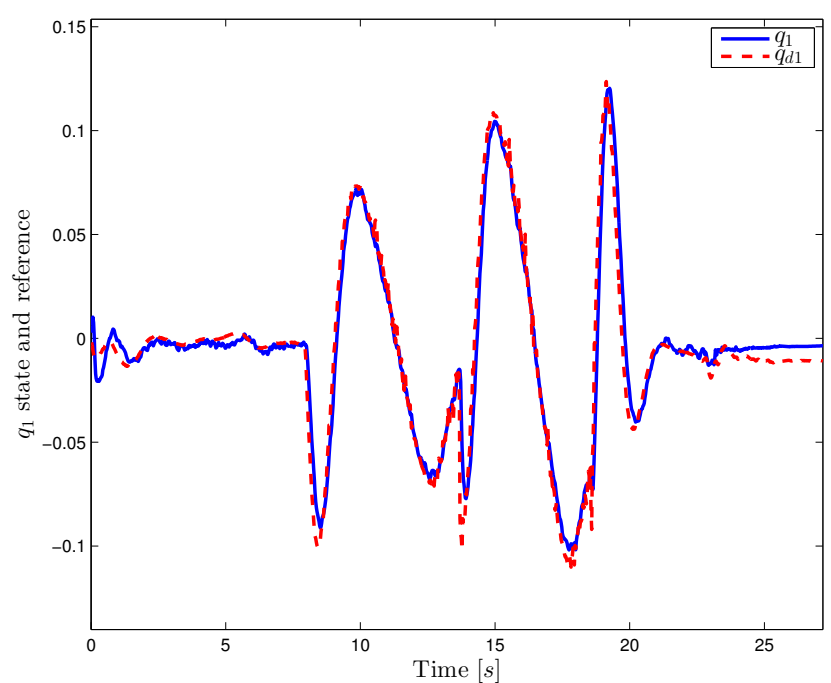

Fig. 36 Attitude and reference for $q_{1}$ in case 1 experiments. 


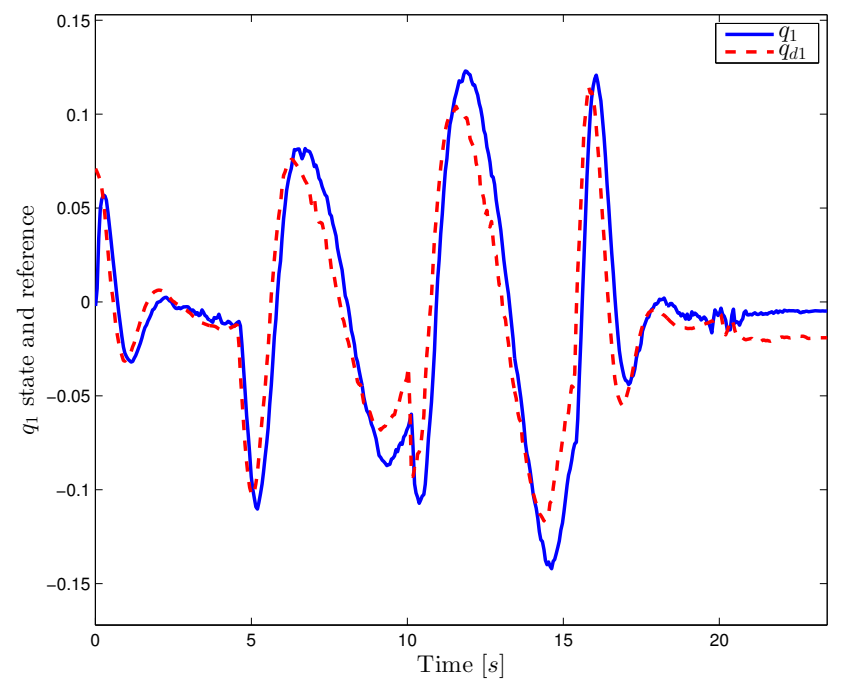

Fig. 37 Attitude and reference for $q_{1}$ in case 2 experiments.

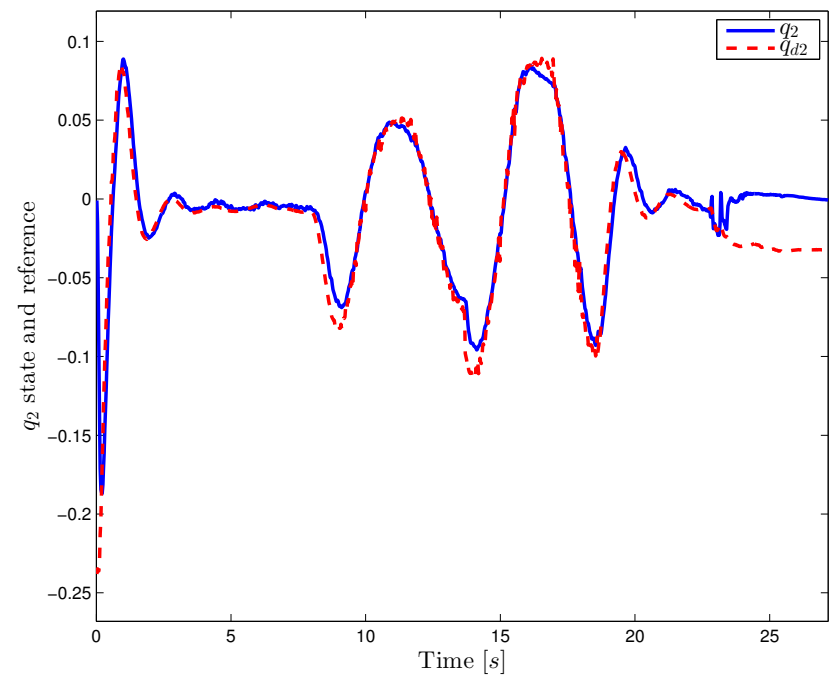

Fig. 38 Attitude and reference for $q_{2}$ in case 1 experiments.

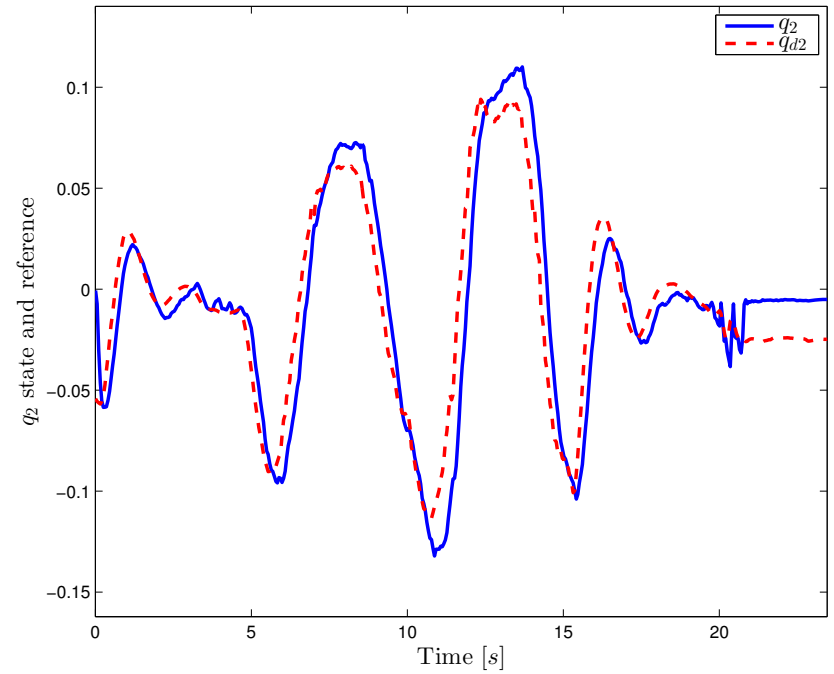

Fig. 39 Attitude and reference for $q_{2}$ in case 2 experiments.
The equivalent Euler Angles were obtained using equation (35), and depicted in Figures 40 to 45.

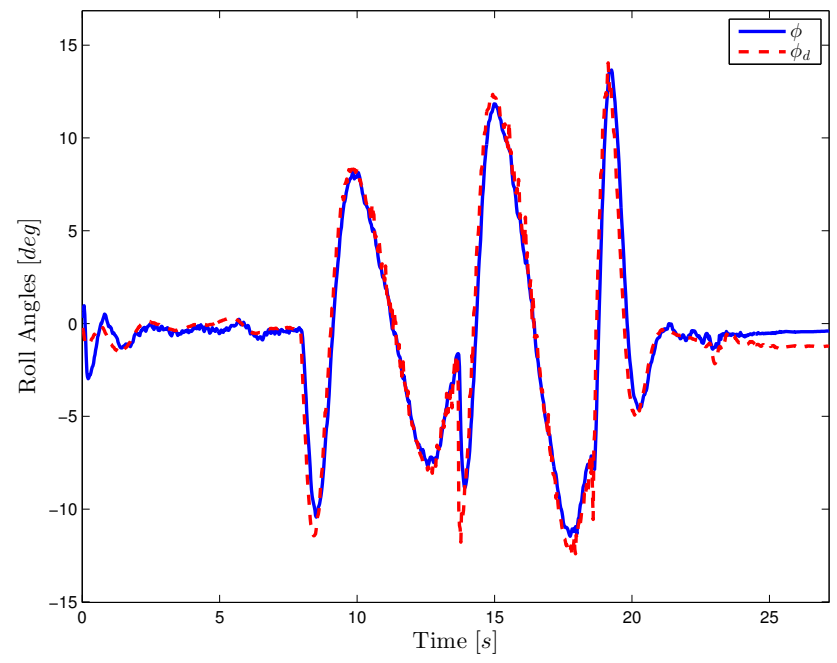

Fig. $40 \phi$ angles in case 1 experiments.

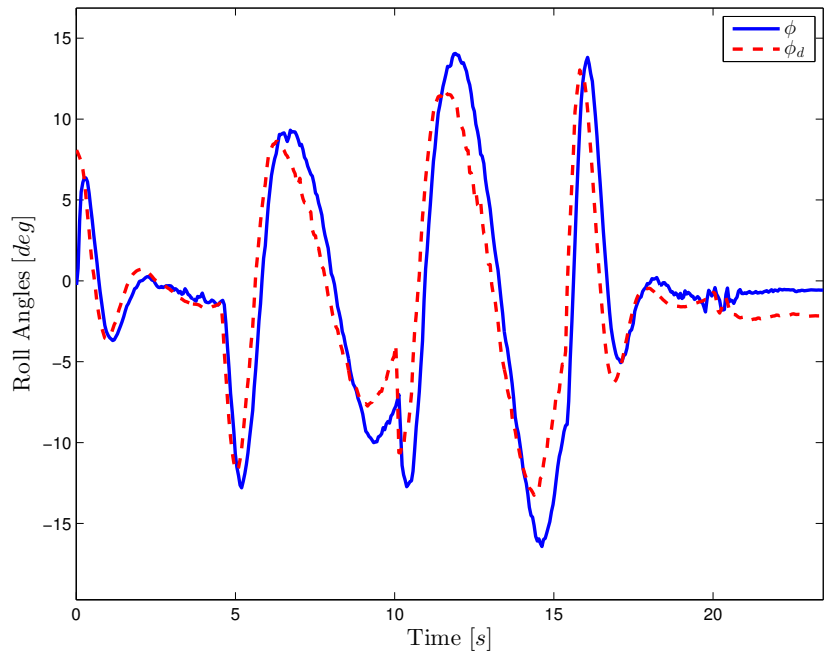

Fig. $41 \phi$ angles in case 2 experiments.

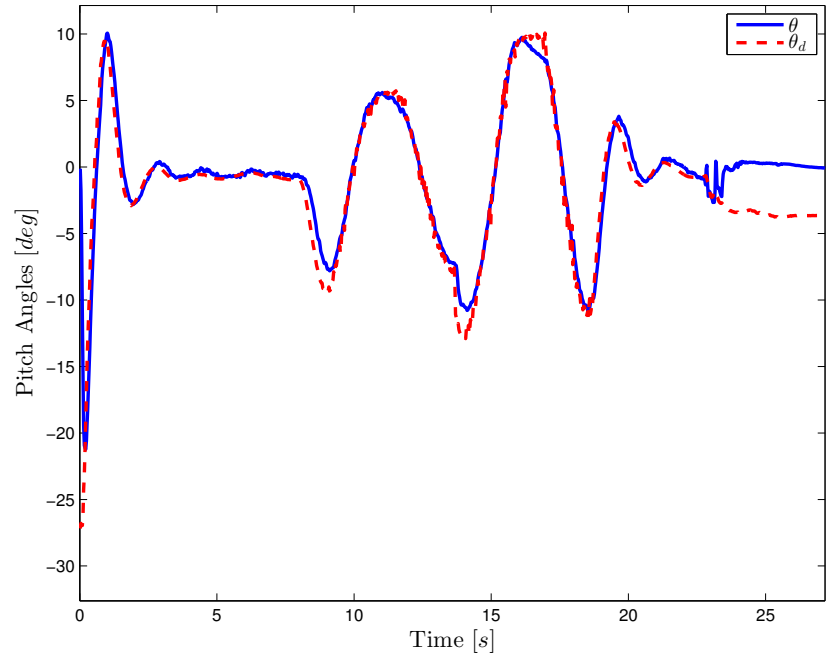

Fig. $42 \theta$ angles in case 1 experiments. 


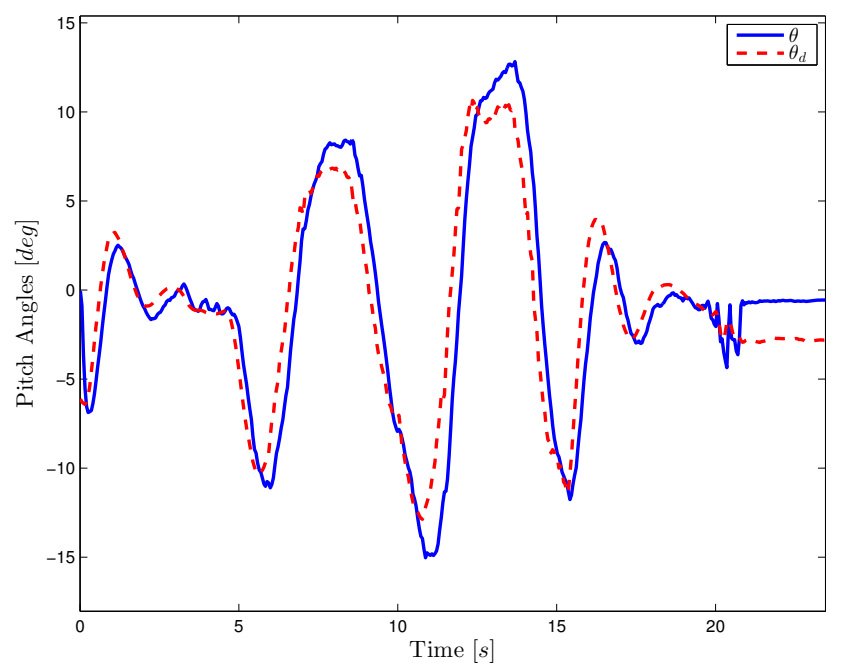

Fig. $43 \theta$ angles in case 2 experiments.

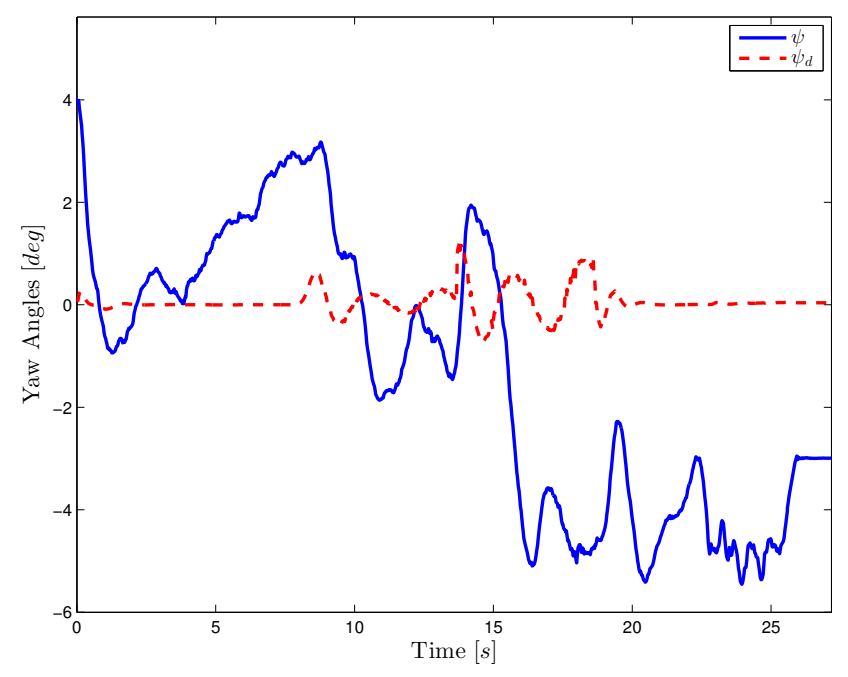

Fig. $44 \psi$ angles in case 1 experiments.

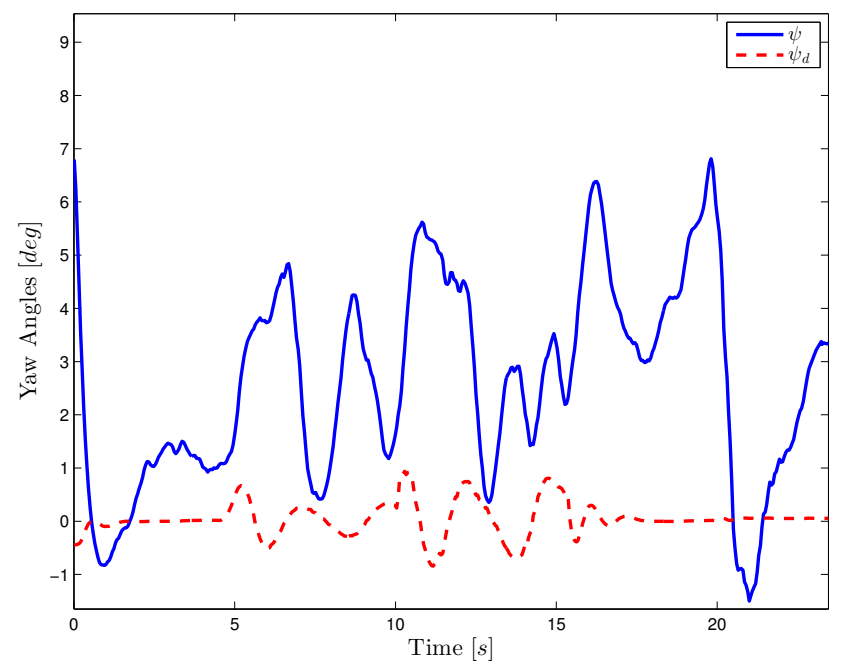

Fig. $45 \psi$ angles in case 2 experiments.
The torques used to control the quad-rotor's attitude are illustrated in Figures 46 and 47, while Figures 48 through 53 represent the position of the vehicle following the desired trajectory.

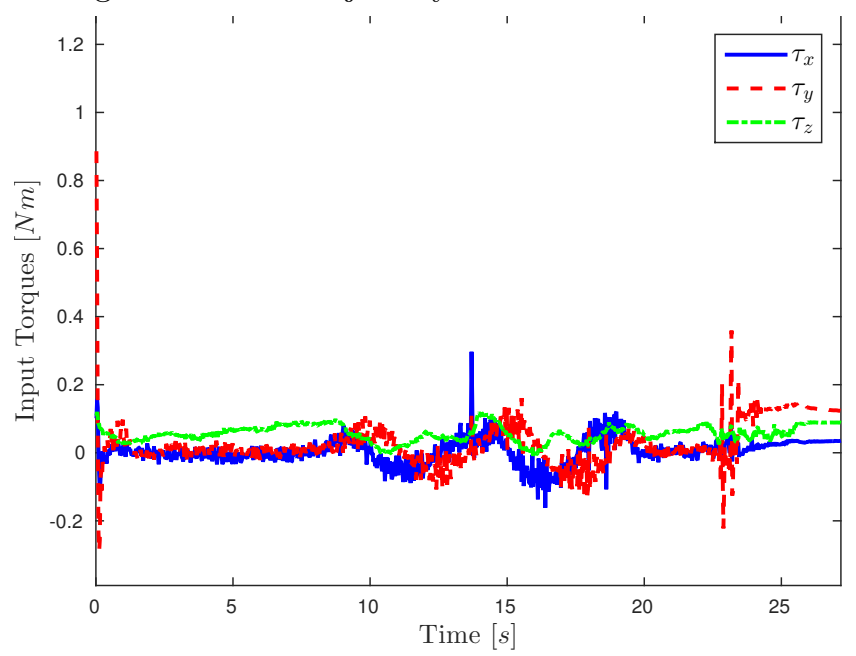

Fig. 46 Quad-rotor's experiment control torques for case 1.

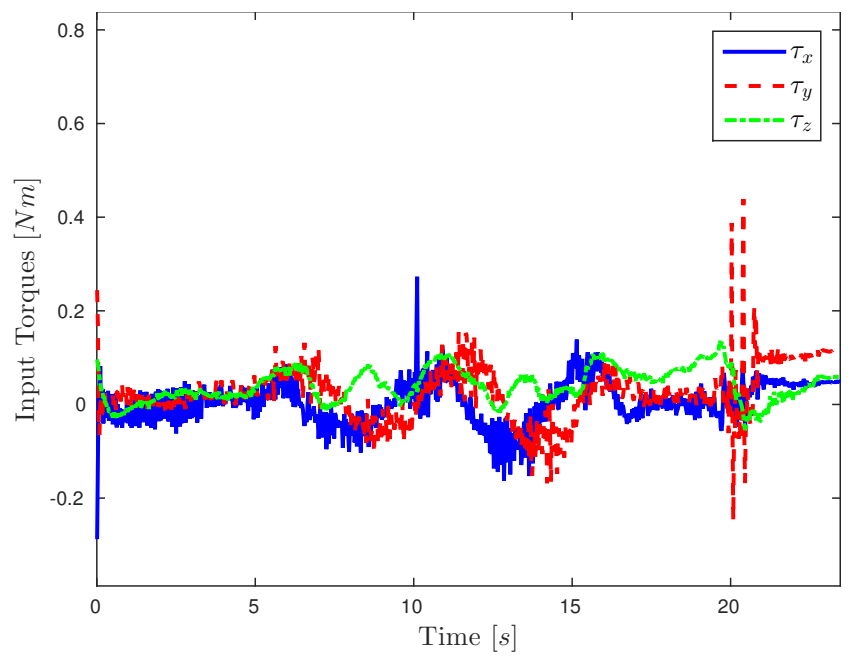

Fig. 47 Quad-rotor's experiment control torques for case 2.

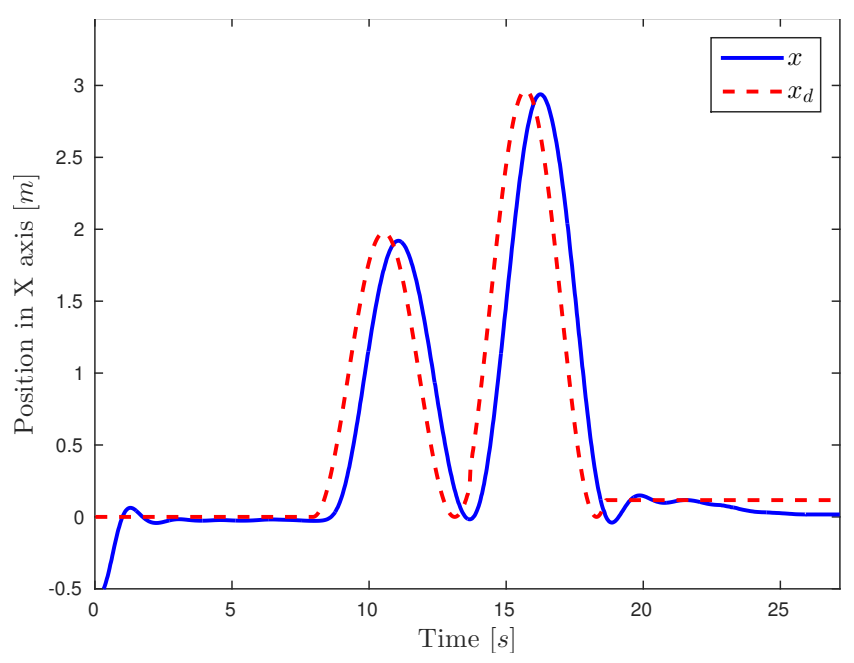

Fig. 48 Quad-rotor's position in the $x$ axis in case 1. 


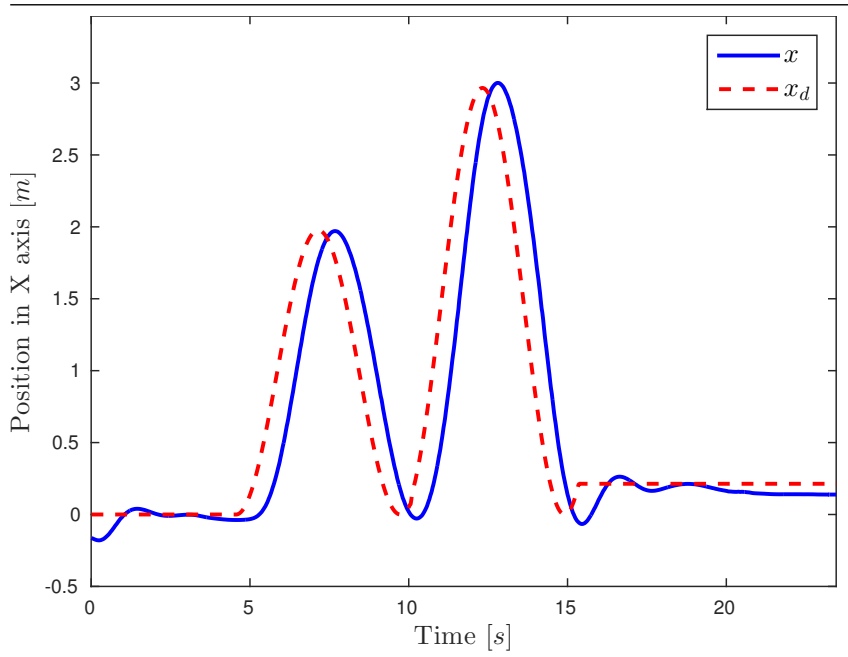

Fig. 49 Quad-rotor's position in the $x$ axis in case 2.

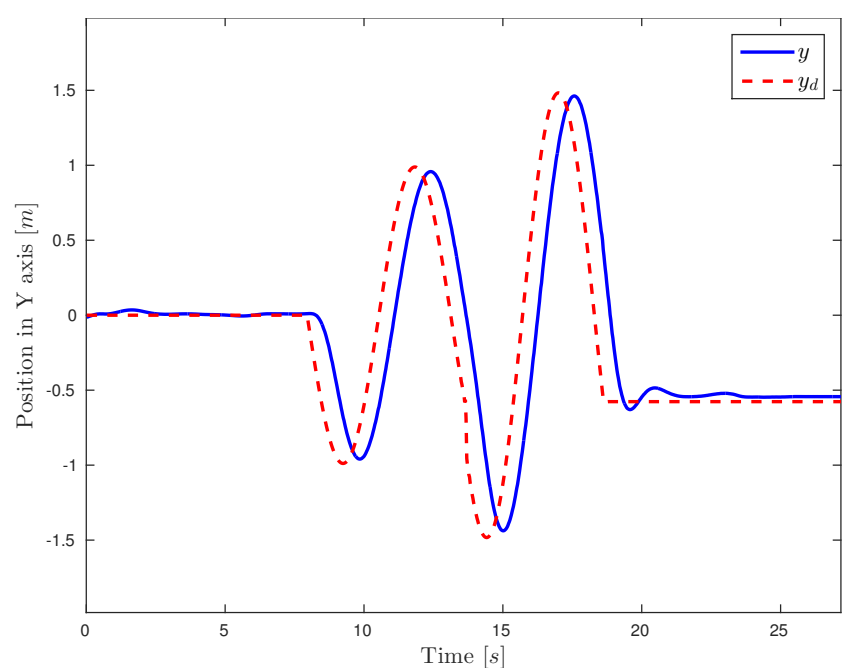

Fig. 50 Quad-rotor's position in the $y$ axis in case 1 .

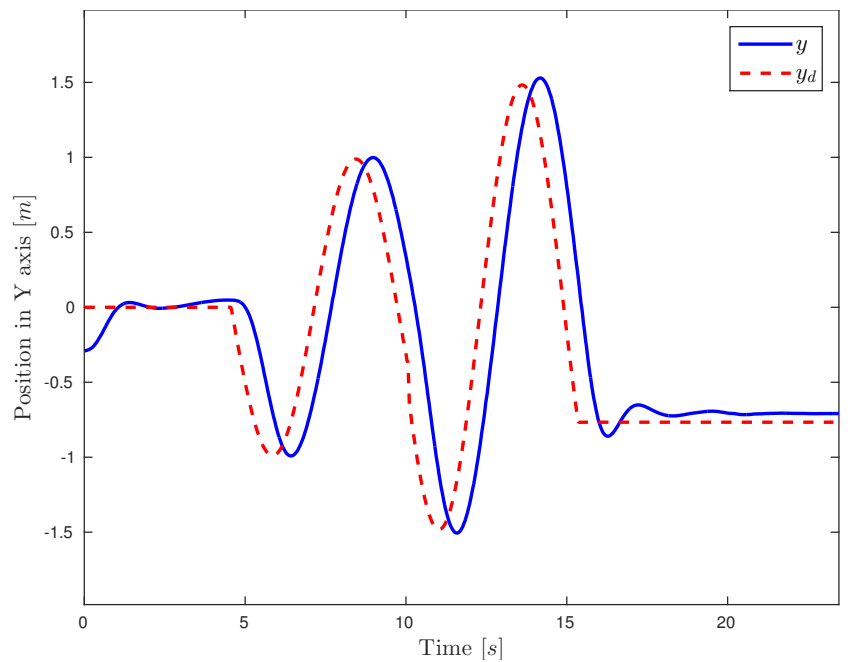

Fig. 51 Quad-rotor's position in the $y$ axis in case 2.

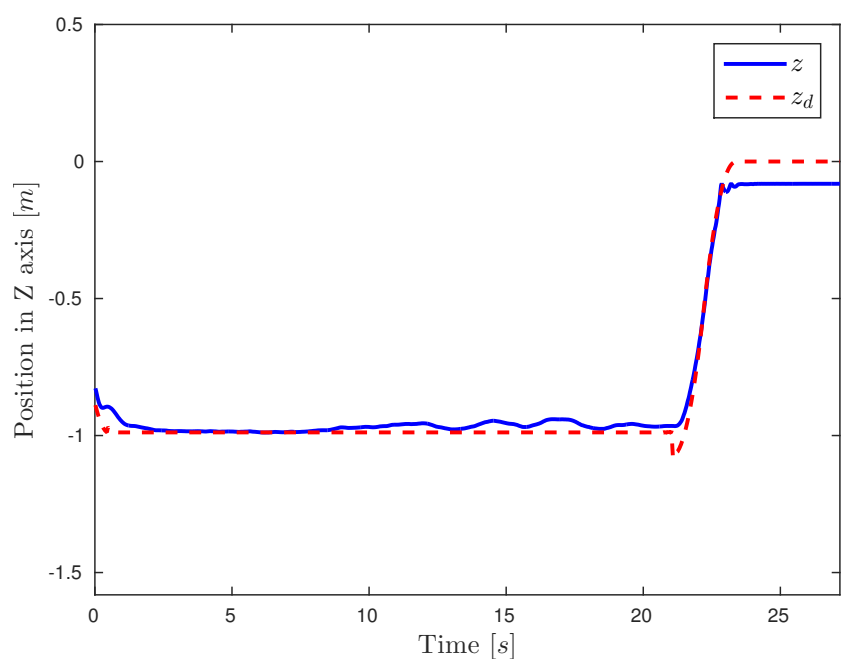

Fig. 52 Quad-rotor's position in the $z$ axis in case 1.

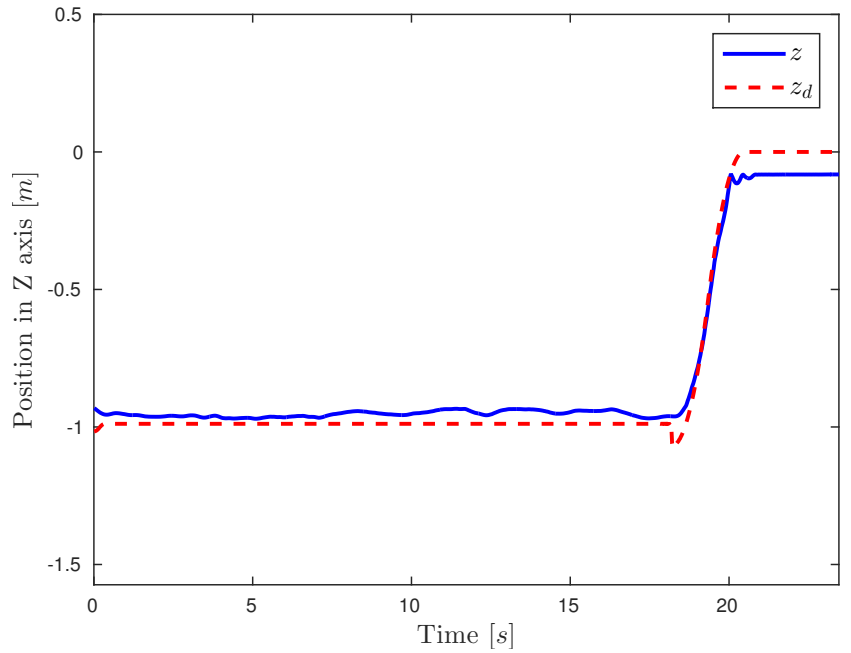

Fig. 53 Quad-rotor's position in the $z$ axis in case 2 .

\subsection{Perturbed flights}

To further validate our proposal, additional flight tests were added with significant perturbations. The UAV was set to follow a $r=1 m$ circular path, then a member of our team pushed the quad-rotor by hand, this push deviates the vehicle from its trajectory. The control laws manage to compensate the disturbance and return the drone to its path.

Figures 54 and 55 illustrate the desired trajectory and the disturbed path taken by the quad-rotor.

The control forces that compensate the perturbations and stabilize the vehicle are depicted in Figures 56 and 57. The quaternion orientation trajectory and the UAV's attitude are compared in Figures 58 through 
69. Note the desired attitude reference adjusts when a disturbance is presented.

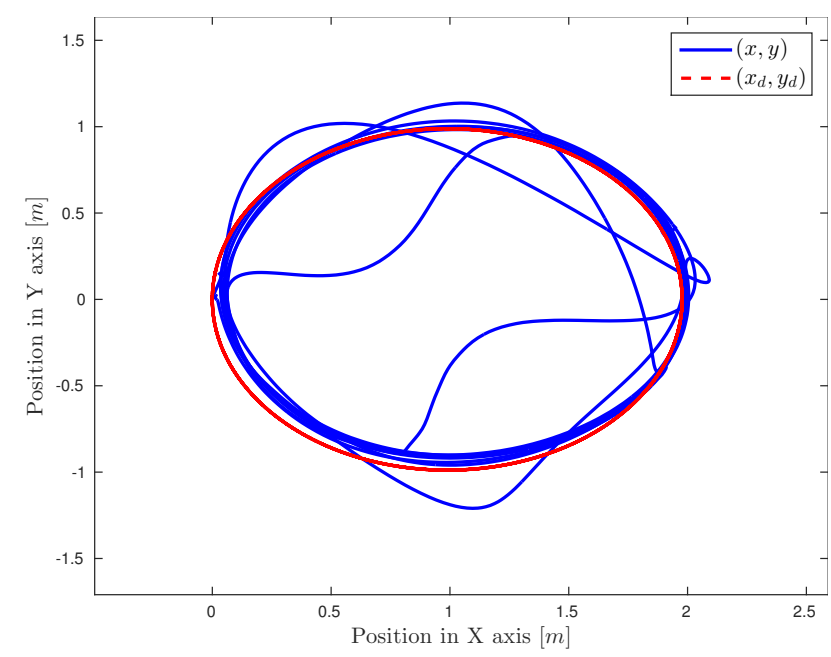

Fig. 54 Quad-rotor's horizontal translation in case 1.

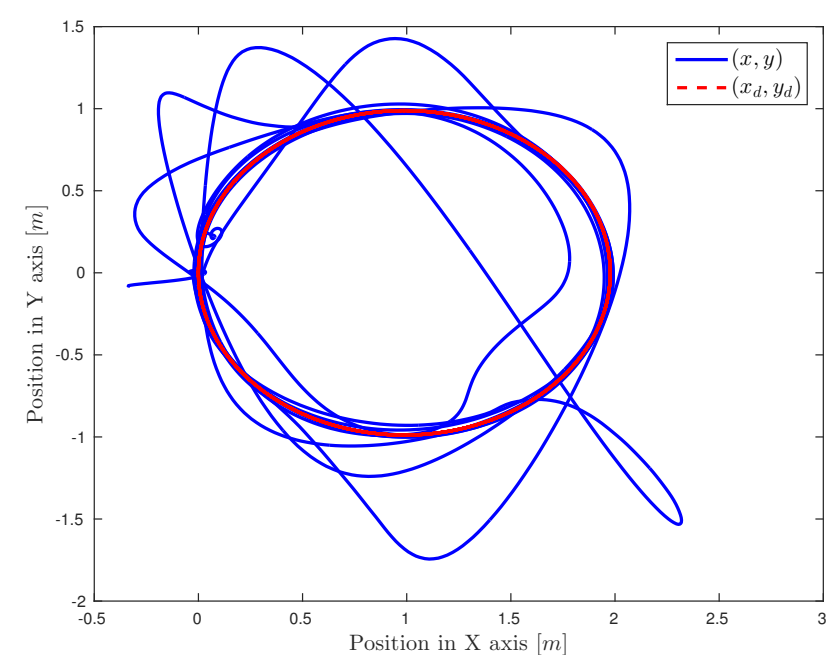

Fig. 55 Quad-rotor's horizontal translation in case 2.

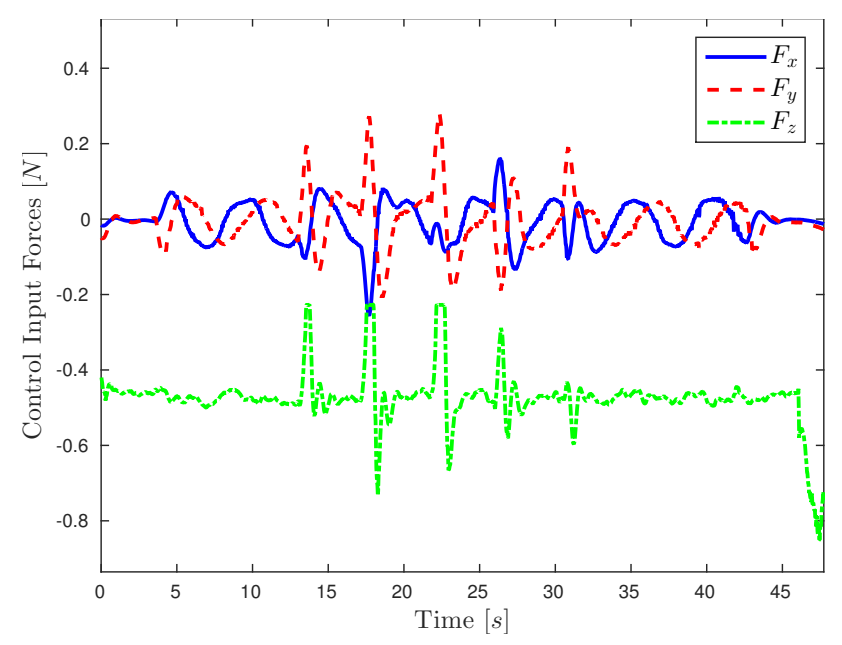

Fig. 56 Quad-rotor's control forces for case 1.

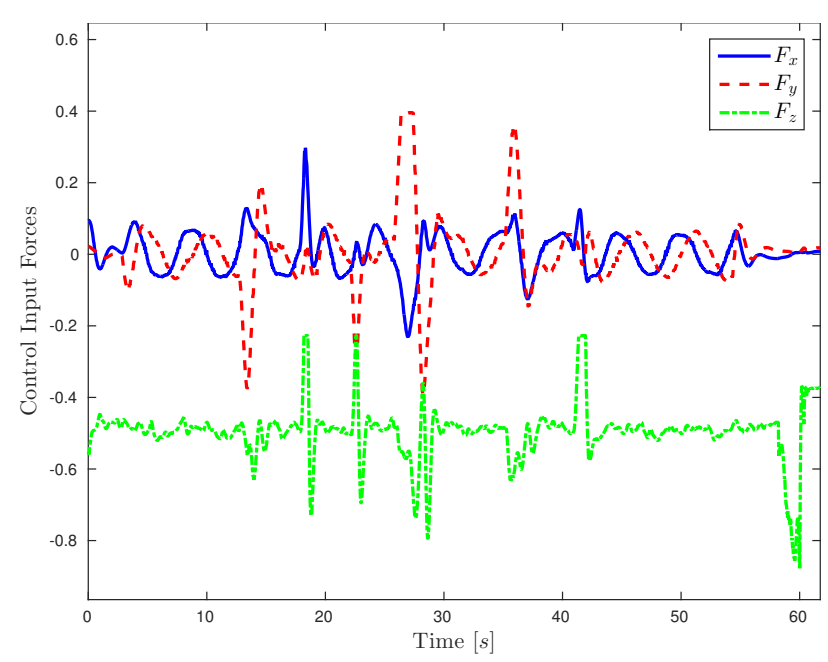

Fig. 57 Quad-rotor's control forces for case 2.

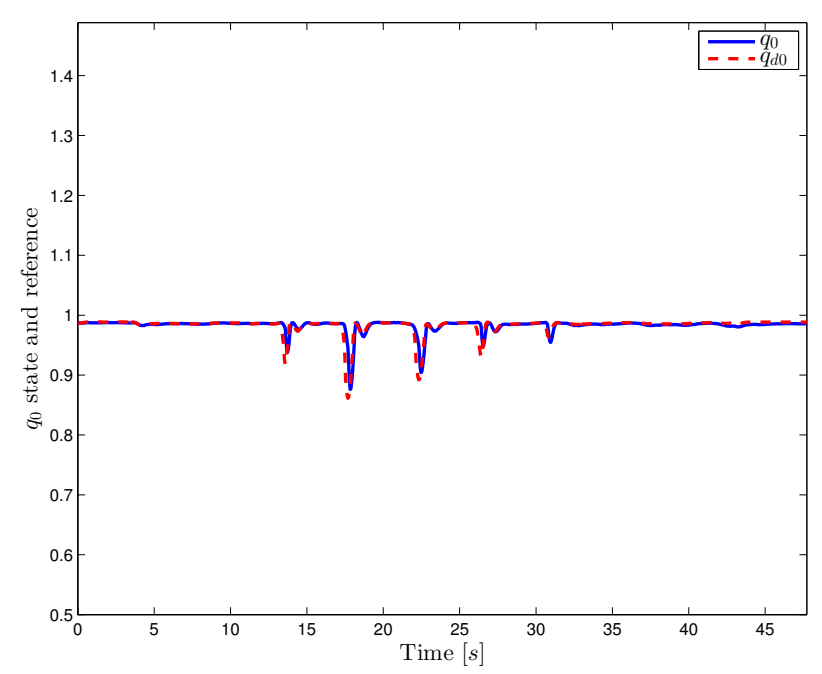

Fig. 58 Attitude and reference for $q_{0}$ in case 1 experiments.

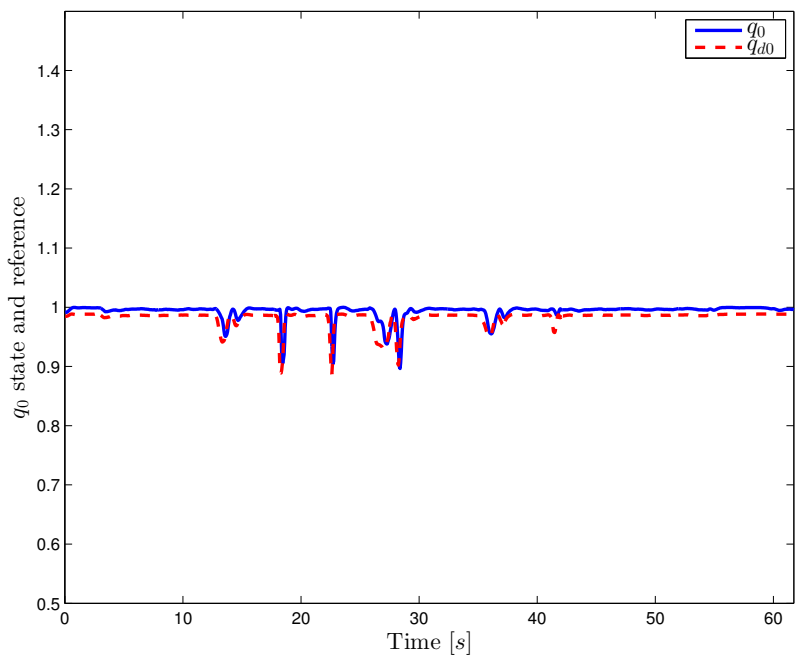

Fig. 59 Attitude and reference for $q_{0}$ in case 2 experiments. 


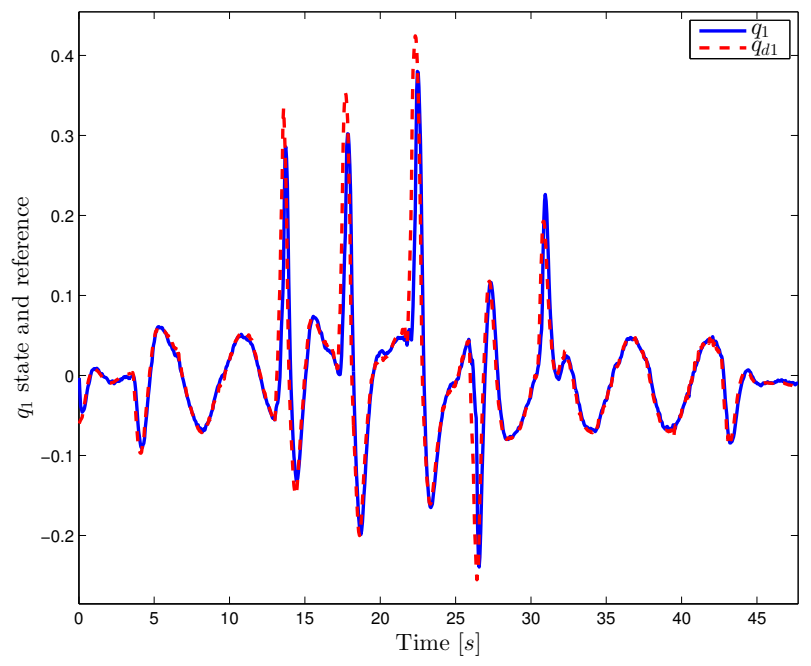

Fig. 60 Attitude and reference for $q_{1}$ in case 1 experiments.

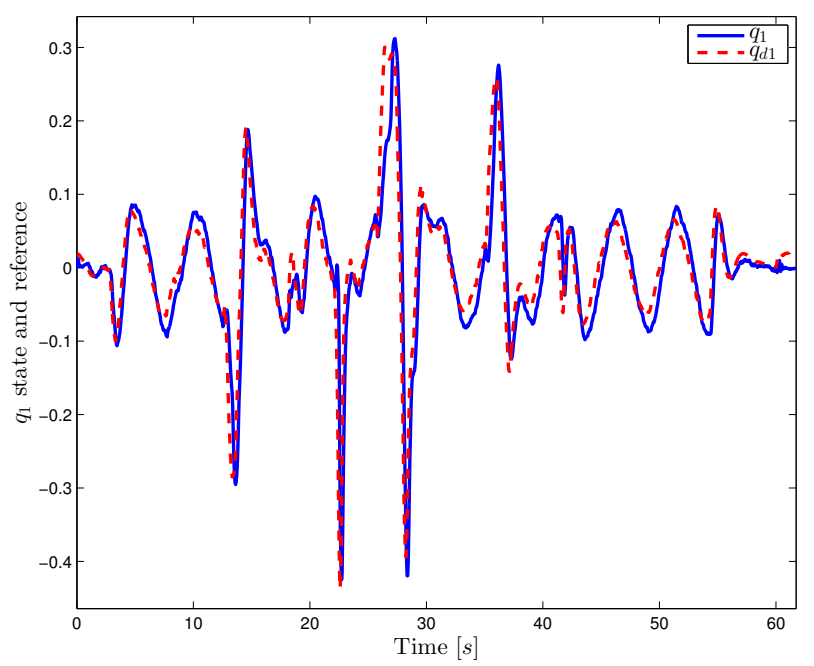

Fig. 61 Attitude and reference for $q_{1}$ in case 2 experiments.

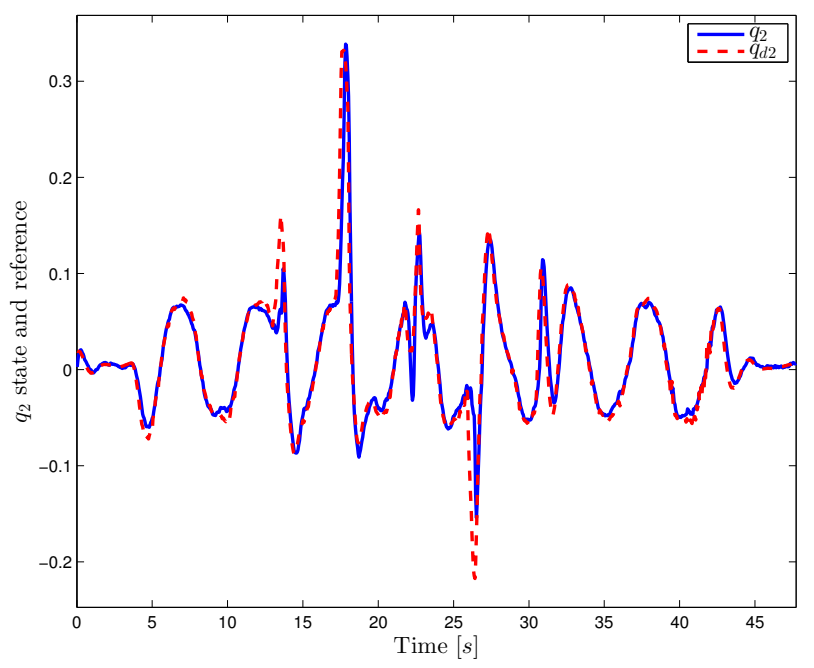

Fig. 62 Attitude and reference for $q_{2}$ in case 1 experiments.
Following equation (35), the Euler angles were computed and illustrated in Figures 63 to 68.

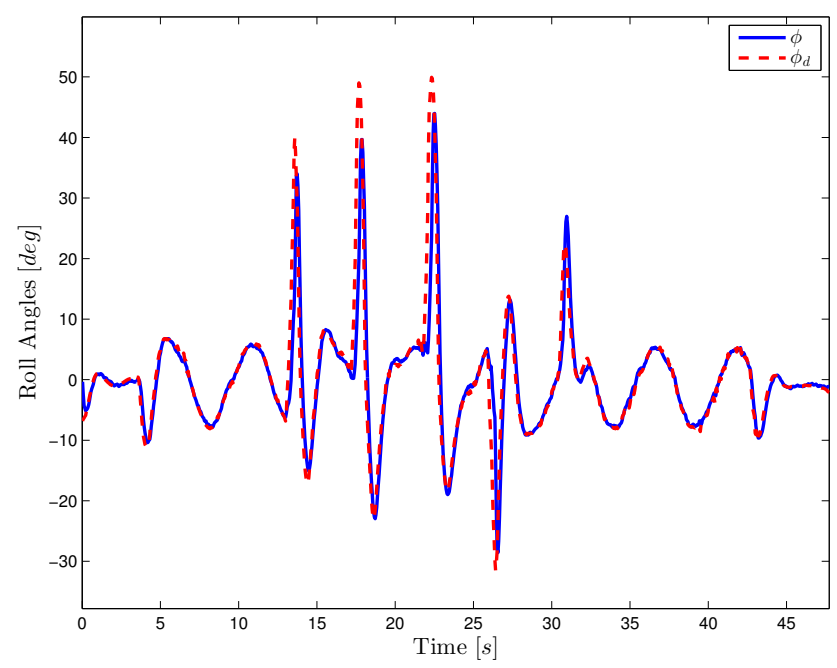

Fig. $63 \phi$ angles in case 1 experiments.

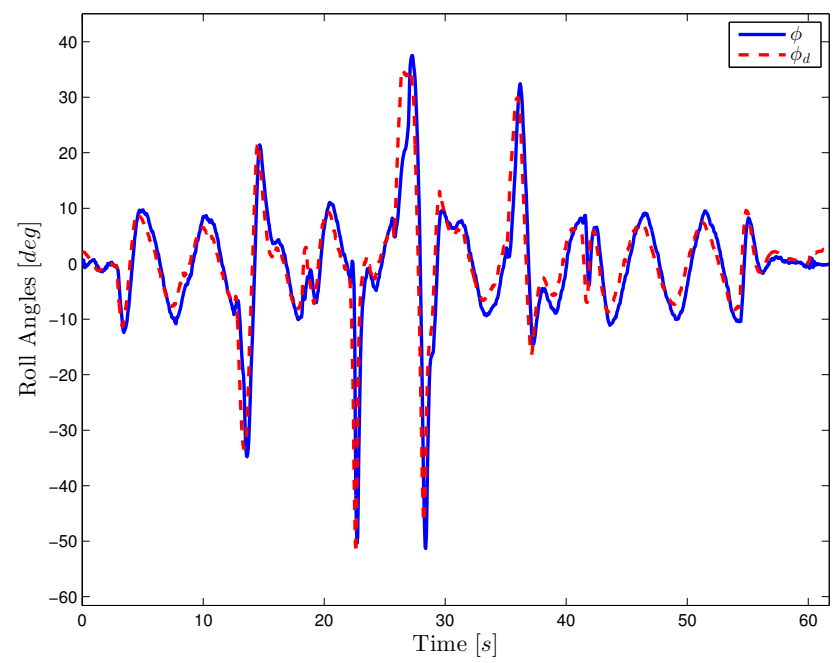

Fig. $64 \phi$ angles in case 2 experiments.

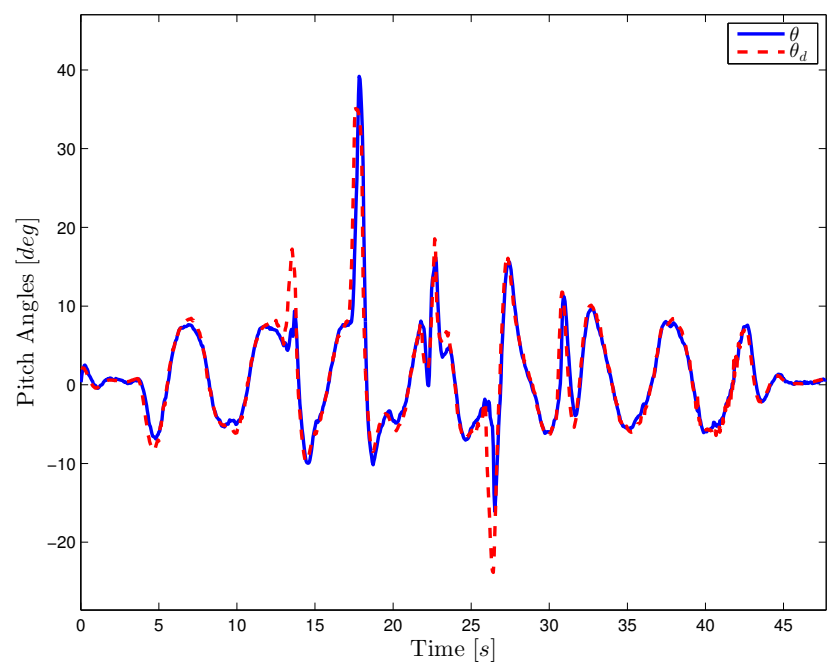

Fig. $65 \theta$ angles in case 1 experiments. 


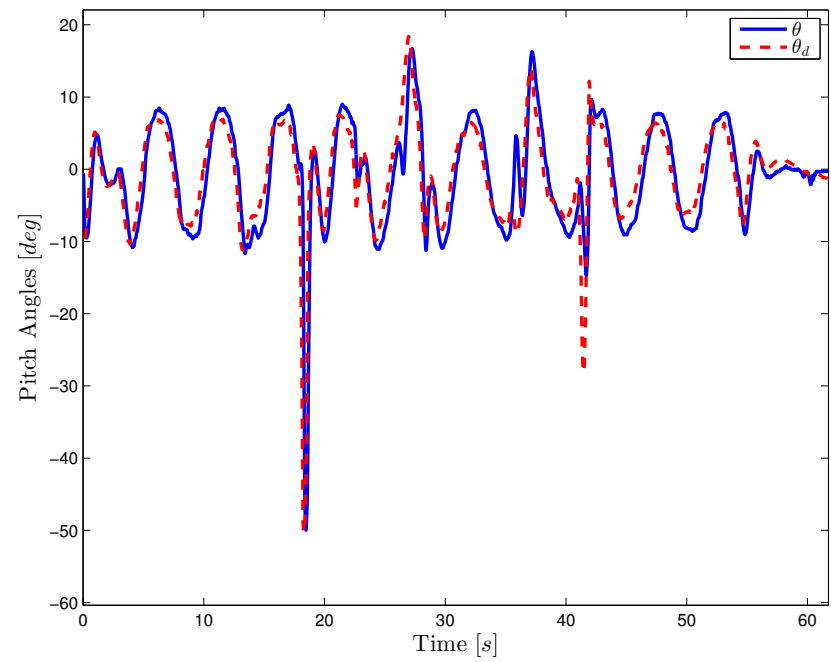

Fig. $66 \theta$ angles in case 2 experiments.

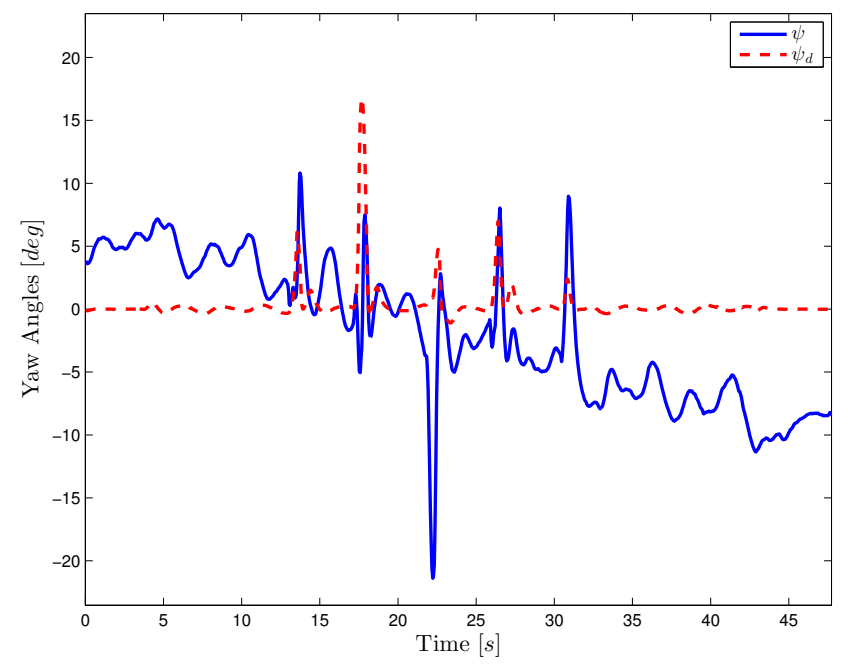

Fig. $67 \psi$ angles in case 1 experiments.

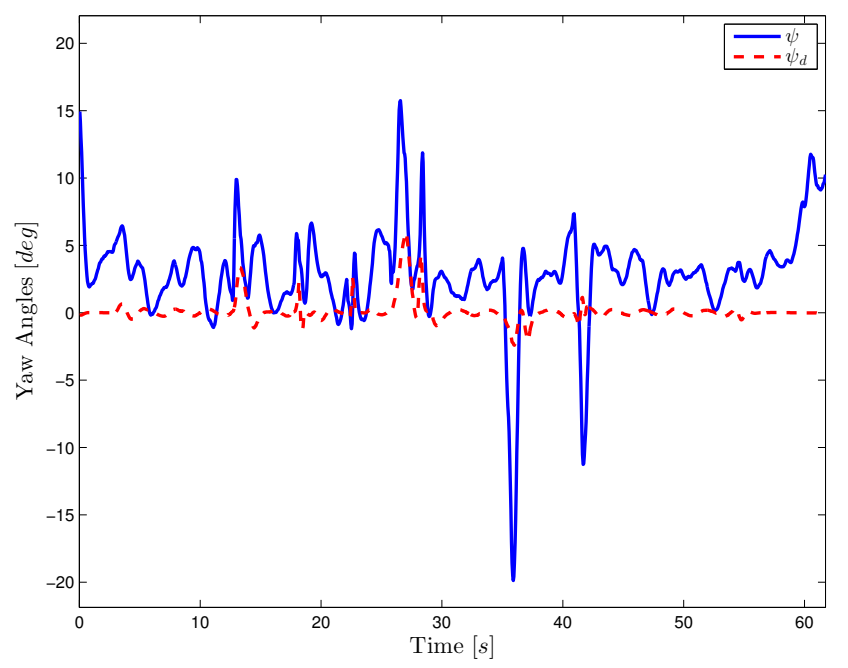

Fig. $68 \psi$ angles in case 2 experiments.

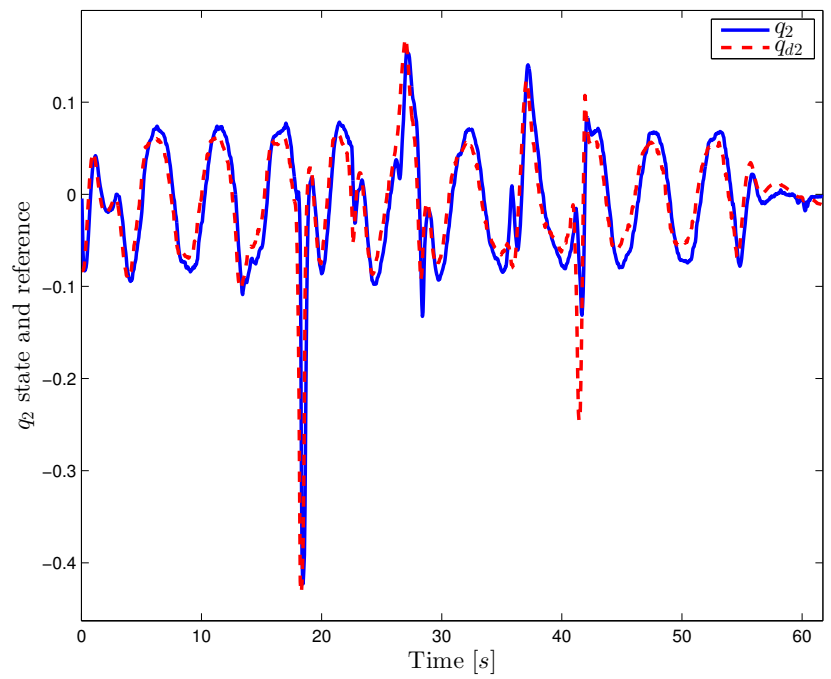

Fig. 69 Attitude and reference for $q_{2}$ in case 2 experiments.

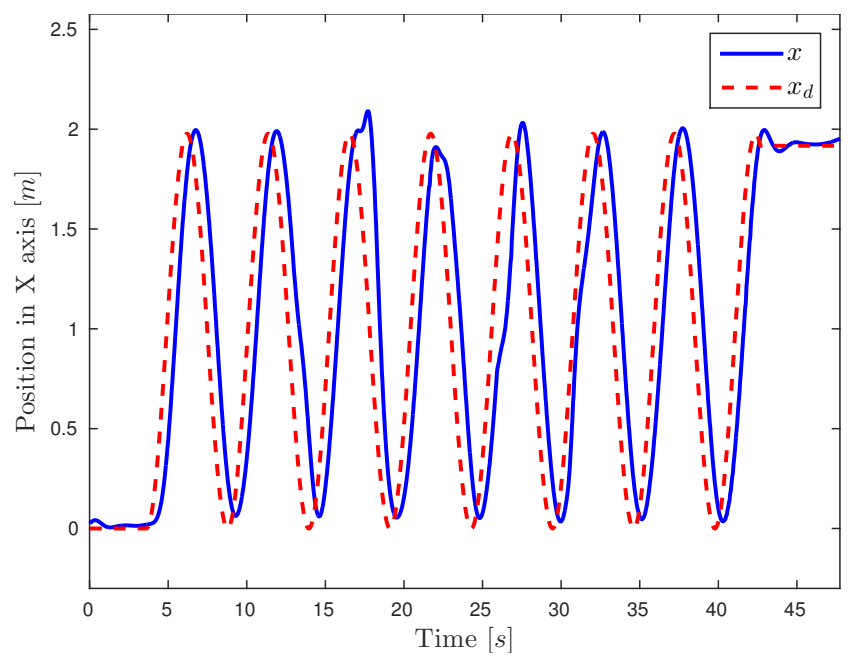

Fig. 70 Quad-rotor's position in the $x$ axis in case 1.

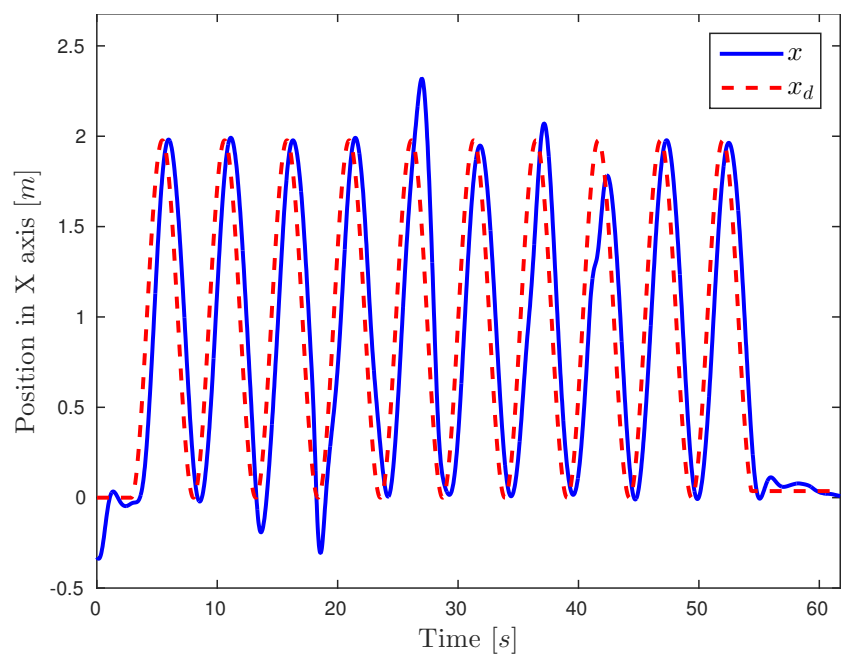

Fig. 71 Quad-rotor's position in the $x$ axis in case 2. 


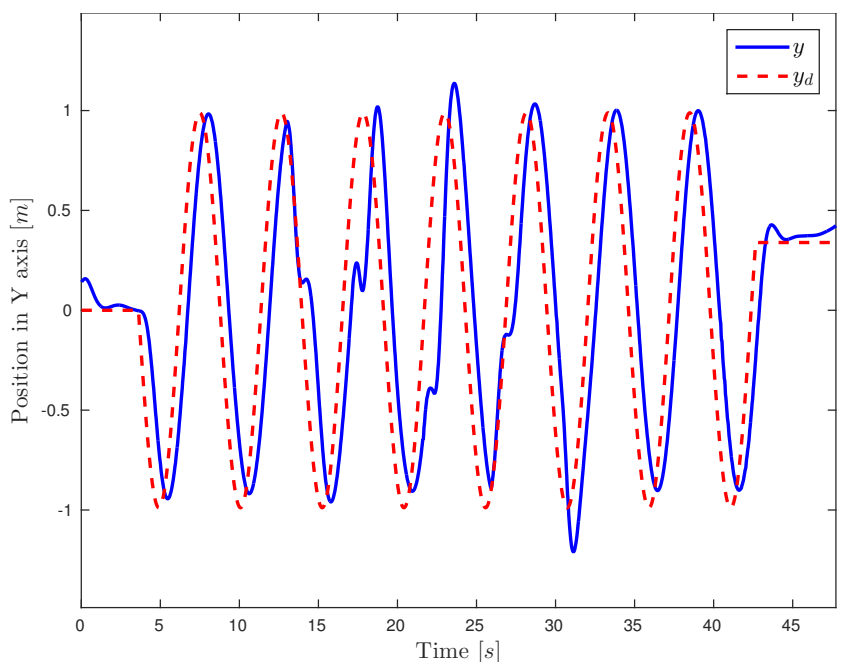

Fig. 72 Quad-rotor's position in the $y$ axis in case 1.

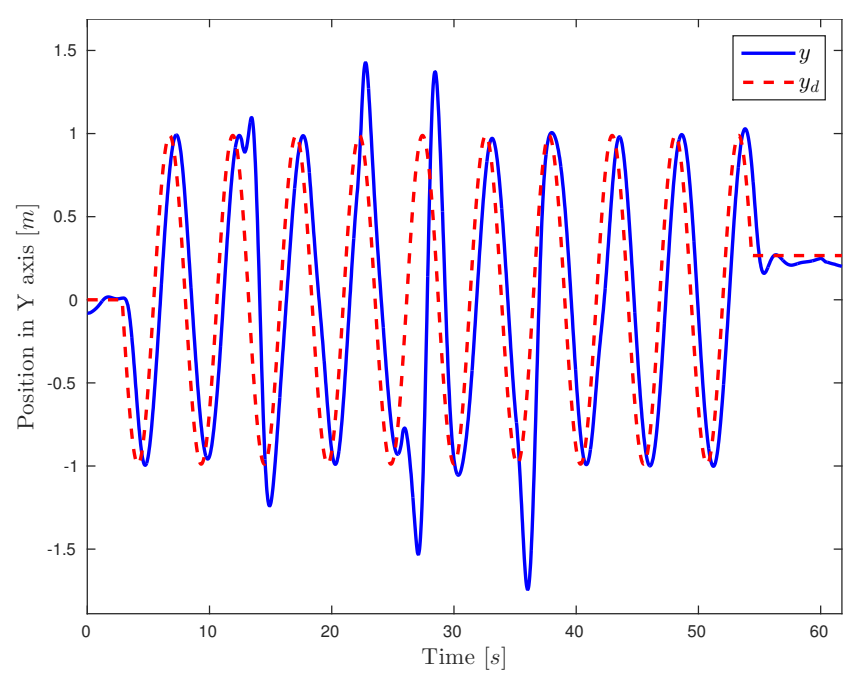

Fig. 73 Quad-rotor's position in the $y$ axis in case 2.

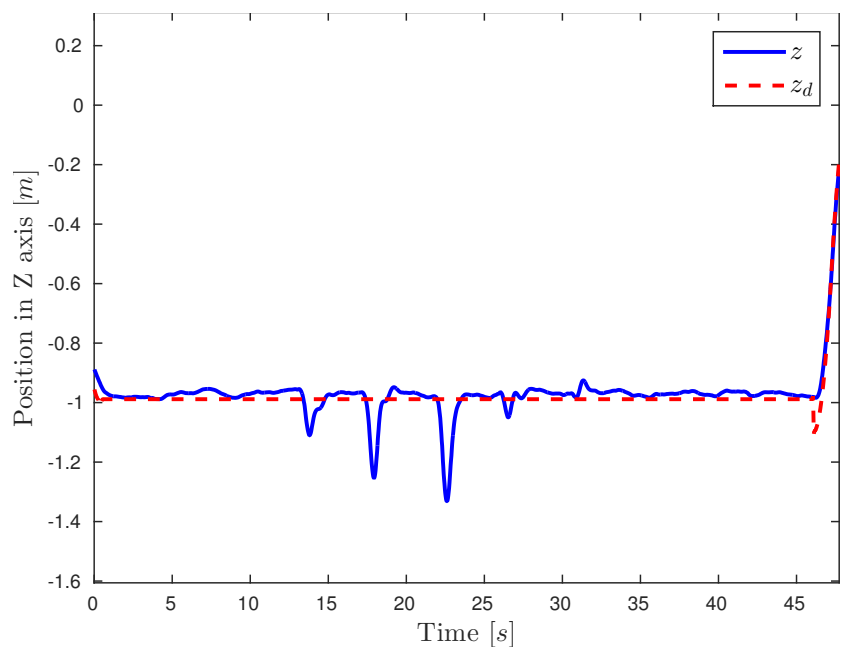

Fig. 74 Quad-rotor's position in the $z$ axis in case 1.

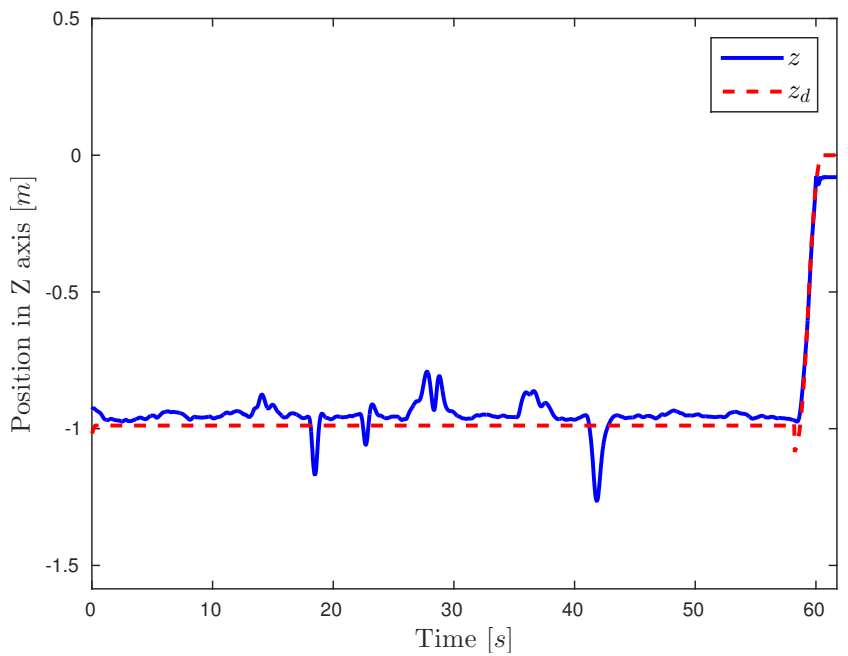

Fig. 75 Quad-rotor's position in the $z$ axis in case 2.

The described experiments were recorded in a video that can be watched in the following link: https://youtu.be/z35Ti_wLRro

\section{CONCLUSIONS}

In this article, the design of a dynamical model based on Euler-Lagrange formalism using a logarithmic mapping in the quaternion space was introduced. The vehicle attitude is denoted by the axis-angle representation of an unit quaternion. The obtained mathematical model through force $\bar{F}_{t h}$ rotated facilitated the control strategy design.

The presented control methods were used to design attitude and also position controllers. These are based on a energy function which has been defined as a Lyapunov function. The controllers use the quaternion representation of the attitude. Also, the attitude controllers use the quaternion error to compute desired torques.

The proposed control strategies allow the stabilization of the full quadrotor dynamics. The presented methodology eliminates undesired effects such as the gimballlock or discontinuities, which are common problems using traditional approaches.

Simulations have shown that the performance of the designed algorithms is satisfactory . The presented experiments validate the application of the proposed control laws in a real quadrotor platform with good performance when tracking a desired trajectory and also in presence of disturbances. 
Future works include the design of control laws for a quadrotor transporting a cable-suspended payload.

\section{Acknowledgment}

The authors would like to thank the Mexican National Council for Science and Technology (CONACYT) for their support with the doctoral scholarships program, as well as the French National Network of Robotics Platforms (ROBOTEX).

\section{References}

1. O. Fritsch, P. De Monte, M. Buhl and B. Lohmann, "Quasi-static Feedback Linearization for the Translational Dynamics of a Quadrotor Helicopter", American Control Conference (ACC), pp. 125-130, 2012.

2. K. Djamel, M. Abdellah and A. Benallegue, "Attitude Optimal Backstepping Controller Based Quaternion for a UAV", Hindawi Publishing Corporation Mathematical Problems in Engineering, vol. 2016, Article ID 8573235.

3. A. Chovancová, T. Fico, P. Hubinský and F. Duchon, "Comparison of various quaternion-based control methods applied to quadrotor with disturbance observer and position estimator", IEEE Robotics and Autonomous Systems, vol. 79, pp. 87-98, 2016.

4. A. Tayebi and S. McGilvray, "Attitude Stabilization of a VTOL Quadrotor Aircraft", IEEE Transactions on Control Systems Technology, vol. 14, no. 3, pp. 562-571, 2006.

5. A. Sanchez, V. Parra-Vega, O. Garcia, F. Ruiz-Sanchez and L.E. Ramos-Velasco, "Time-Parametrization Control of Quadrotors with a Robust Quaternion-based Sliding Mode Controller for Aggressive Maneuvering", European Control Conference (ECC), pp. 3876-3881, Zurich, Switzerland, 2013.

6. E. Fresk and G. Nikolakopoulos, "Full Quaternion Based Attitude Control for a Quadrotor", IEEE European Control Conference $(E C C)$, Zurich, Switzerland, 2013.

7. J. Cariño, H. Abaunza and P. Castillo. "Quadrotor Quaternion Control", International Conference on Unmanned Aircraft Systems (ICUAS), Denver, USA, 2015.

8. R. Dargham and H. Medromi. "Euler and Quaternion Parameterization in VTOL UAV Dynamics with Test Model Efficiency", International Journal of Applied Information Systems (IJAIS), vol. 9, no. 8, 2015.

9. A. Honglei, L. Jie, W. Jian, W. Jianwen and M. Hongxu, "Backstepping-Based Inverse Optimal Attitude Control of Quadrotor", International Journal of Advanced Robotic Systems, DOI: 10.5772/56337, vol. 10, 2013.

10. A.A. El-Badawy and M.A. Bakr, "Quadrotor Aggressive Maneuvers along Singular Configurations: An EnergyQuaternion Based Approach", Journal of Control Science and Engineering, ID 7324540, 2016.

11. O. Fritsch, D. Tromba, and B. Lohmann "Cascaded energy based trajectory tracking control of a quadrotor", Automatisierungstechnik, vol. 62, no. 6, pp. 408-422, 2014.

12. M.E. Guerrero, R. Lozano and C.D. García, "Control Basado en Pasividad para un quadrotor UAV", IEEE Congreso Nacional de Control Automático (AMCA), Morelos, Mexico, 2015.
13. C. Souza, G.V. Raffo and E.B. Castelan, "Passivity Based Control of a Quadrotor", 19th World Congress The International Federation of Automatic Control (IFAC) Cape Town, South Africa, pp. 24-29, 2014.

14. L.E. Muñoz, O. Santos, P. Castillo and I. Fantoni, "Energy-based nonlinear control for a quadrotor rotorcraft", American Control Conference (ACC), pp. 11771182, Washington, DC, USA, 2013.

15. N. Kottenstette and J. Porter, "Digital Passive Attitude and Altitude Control Schemes for Quadrotor Aircraft", IEEE International Conference on Control and Automation (ICCA), Christchurch, New Zealand, pp. 1761-1768, 2009.

16. K.W Spring. "Euler parameters and the use of quaternion algebra in the manipulation of finite rotations: a review". Mechanism and Machine Theory, vol. 21, no. 5 pp. 365373, 1986.

17. S.L. Altmann, "Hamilton, Rodrigues, and the quaternion scandal". Mathematics Magazine, pp. 291-308, 1989.

18. R. Campa and K. Camarillo, "Unit quaternions: A mathematical tool for modeling, path planning and control of robot manipulators", Robot manipulators, M. Ceccarelli (ed.), In-Teh, pp. 21-48, 2008.

19. J.B Kuipers, "Quaternions and rotation sequences", vol. 66. Princeton university press Princeton, 1999. 